\title{
ANALYSIS OF STOCHASTIC FLUID QUEUES DRIVEN BY LOCAL-TIME PROCESSES
}

\author{
TAKIS KONSTANTOPOULOS, ${ }^{*}$ Heriot-Watt University \\ ANDREAS E. KYPRIANOU, ${ }^{* *}$ University of Bath \\ PAAVO SALMINEN $* * * * * * *$ AND \\ MARINA SIRVIÖ, ${ }^{* * *}$ Åbo Akademi University
}

\begin{abstract}
We consider a stochastic fluid queue served by a constant rate server and driven by a process which is the local time of a reflected Lévy process. Such a stochastic system can be used as a model in a priority service system, especially when the time scales involved are fast. The input (local time) in our model is typically (but not necessarily) singular with respect to the Lebesgue measure, a situation which, in view of the nonsmooth or bursty nature of several types of Internet traffic, is nowadays quite realistic. We first discuss how to rigorously construct the (necessarily) unique stationary version of the system under some natural stability conditions. We then consider the distribution of performance steady-state characteristics, namely, the buffer content, the idle period, and the busy period. These derivations are much based on the fact that the inverse of the local time of a Markov process is a Lévy process (a subordinator), hence making the theory of Lévy processes applicable. Another important ingredient in our approach is the use of Palm calculus for stationary random point processes and measures.
\end{abstract}

Keywords: Local time; fluid queue; Lévy process; Skorokhod reflection; performance analysis; Palm calculus; inspection paradox

2000 Mathematics Subject Classification: Primary 60G10; 60G55; 60G51; 90B15

\section{Introduction}

This paper extends the results of Mannersalo et al. [20] who introduced a fluid queue (or storage process) driven by the local time at 0 of a reflected Brownian motion and served by a deterministic server with constant rate. The motivation provided in [20] was that the system provides a macroscopic view of a priority queue with two priority classes. Indeed, in such a system, the highest priority class (class 1) goes through as if the lowest one does not exist, whereas the lowest priority class (class 2) gets served whenever no item of the highest priority is present. In telecommunications terminology, class 2 receives only whatever bandwidth remains after class 1 is served. As argued in [20], if the highest priority queue is, macroscopically, approximated by a reflected Brownian motion, the lowest priority queue is driven by the cumulative idle time of the first one, which is approximated by the local time of the reflected Brownian motion at 0 .

Received 14 September 2007; revision received 14 August 2008.

* Postal address: School of Mathematical and Computer Sciences, Heriot-Watt University, Edinburgh EH14 4AS, UK.

** Postal address: Department of Mathematical Sciences, University of Bath, Bath BA2 7AY, UK.

*** Postal address: Department of Mathematics, Åbo Akademi University, Turku, FIN-20500, Finland.

**** Email address: phsalmin@abo.fi 
One motivation for the study of our stochastic model is due to the so-called Internet neutrality issue. Internet neutrality is the principle that all content transmitted over a network be treated equally and without preference. However, because of many factors involved (such as the fact that more than $40 \%$ of Internet traffic is due to peer-to-peer networks, and, mostly, BitTorrent [6]), it is quite likely that flow classification and differential classes of service will be introduced. In view of this, understanding a two-queue priority service system at a macroscopic level such as ours is important.

From a mathematical point of view, the model is a rare example of a nontrivial fluid queue whose performance characteristics (such as steady-state distribution) can be computed explicitly. If, in addition, we take into account the heavy-tailed nature of traffic on the Internet, it seems reasonable to consider a Lévy process as a model for class 1 queues. This provides the motivation for studying a queue whose input is the local time of a reflected Lévy process.

More generally, let $X$ be a Markov process and let $L$ be its local time at a specific point. The fluid queue driven by $X$ refers to the stochastic system defined by

$$
Q_{t}=Q_{0}+L_{t}-t+I_{t}, \quad t \geq 0,
$$

where $Q_{t} \geq 0$ for all $t \geq 0$ and $I$ is a nondecreasing process, starting from 0 , such that

$$
\int_{0}^{\infty} \mathbf{1}\left(Q_{s}>0\right) \mathrm{d} I_{s}=0 .
$$

It is known (see [15]) that (1) and (2) imply that $I$ is given by

$$
I_{t}=-\inf _{0 \leq s \leq t}\left\{\left(Q_{0}+L_{s}-s\right) \wedge 0\right\} .
$$

We say that $Q$ is obtained by Skorokhod reflection. By considering, instead of 0 , an arbitrary initial time, we can define a proper stochastic dynamical system (see Appendix A for details) which, under natural conditions, admits a unique stationary version. We refer frequently to this throughout the paper.

We also remark that in Kozlova and Salminen [17] the situation in which $X$ is a general onedimensional diffusion is analysed. Moreover, Sirviö (née Kozlova) [23] studied the case where $L$ is constructed as the inverse of a general subordinator (without specialising the underlying process $X$ ). Also, there are papers in the literature where reflections of drifted local-time processes have been considered, rather implicitly; for example, in a network of fluid queues, the content of one queue is influenced by the reflector (which coincides in some cases with our notion of local time) of an upstream queue. See, e.g. [13] for such a case: the process $I_{2}$ of [13, Equation (2.8)] is closely related to our process $Q$, but in that paper, the process $I_{2}$ is not the per se object of study. (See also [11] for a tandem fluid network case and, of course, the rather rich literature of Brownian fluid networks; see, e.g. [8] and the references therein.)

In this paper we follow ideas which were developed in [23] in the context of reflection of the inverse of a subordinator. However, (i) we connect the abstract framework with the case where the subordinator is the local time of a reflected Lévy process (motivated by applications in priority processing systems), (ii) we use, as much as possible, a framework based on Palm probabilities (see, in particular, Theorem 3 and Lemma 2), and (iii) we explicitly discuss the possible types of sample path behaviour of the process of interest $(Q)$; for an illustration, see Figures 1,2, and 3. Figure 1 concerns the case where $Q$ has continuous sample paths but with parts which are singular with respect to the Lebesgue measure. Figure 2 concerns the case where $Q$ has paths with isolated discontinuities (positive jumps) and linear decrease between them. 
Figure 3 concerns the case where $Q$ has absolutely continuous paths. The cases are exhaustive. Despite the wide variety of sample paths (depending on the type of underlying Lévy process $Y$ ), the mathematical framework and formulae derived have a uniform appearance.

The paper is organised as follows. In Section 2 we construct the stationary version of the underlying (background) Markov process $X$. In Section 3 we construct the stationary version of the stochastic fluid queue with input taken as the local time of $X$, based on the stationary version of $X$. In Section 4 we derive the stationary distribution of the buffer content and present a number of examples. In Section 5 we examine the idle and busy periods, and, in particular, characterise the distributions of their starting and ending times. The analysis is carried out first under the condition that the time at which the system is observed is a typical point of an idle or busy period. Finally, the distributions of typical idle and busy periods are derived.

\section{The background Markov process and its local time}

We first construct the underlying Markov process $X$ which models the highest priority class. This process will be taken to be the stationary reflection of a spectrally one-sided Lévy process

$$
Y=\left(Y_{t}, t \in \mathbb{R}\right)
$$

with two-sided time and $Y_{0}=0$ (see Appendix B, where $Y$ is defined on an appropriate probability space $(\Omega, \mathcal{F}, \mathrm{P})$ together with a family of shifts $\left(\theta_{t}, t \in \mathbb{R}\right)$ which will be used throughout the paper). A Lévy process is called spectrally negative if its Lévy measure $\Pi$ satisfies

$$
\Pi((-\infty, 0))>0 \quad \text { and } \Pi((0,+\infty))=0,
$$

and spectrally positive if

$$
\Pi((-\infty, 0))=0 \quad \text { and } \quad \Pi((0,+\infty))>0 .
$$

Clearly, if $Y$ is spectrally positive then $-Y$ is spectrally negative, and vice versa. To avoid trivialities, we shall assume throughout that

$Y$ does not have monotone paths,

which rules out the cases that $Y$ is an increasing or decreasing subordinator.

We also discuss the characteristics of its local time at 0 . In Appendix A we summarise the notation and results on the Skorokhod reflection problem and its stationary solution. In Appendix B we summarise some facts on Lévy processes, indexed by $\mathbb{R}$, with one-sided jumps. We will denote throughout by $\mathrm{P}$ a probability measure which is invariant under time shifts, and we shall let

$$
\mathrm{P}_{x}(\cdot):=\mathrm{P}\left(\cdot \mid X_{0}=x\right),
$$

i.e. the conditional probability $\mathrm{P}$ given $X_{0}$, evaluated at $X_{0}=x$. We define the Laplace exponent of a spectrally one-sided Lévy process as a function $\psi_{Y}: \mathbb{R}_{+} \rightarrow \mathbb{R}$ given, for $\theta \geq 0$, by

$$
\psi_{Y}(\theta):= \begin{cases}\log \mathrm{E}\left[\exp \left\{\theta\left(Y_{t+1}-Y_{t}\right)\right\}\right] & \text { if } Y \text { is spectrally negative } \\ \log \mathrm{E}\left[\exp \left\{-\theta\left(Y_{t+1}-Y_{t}\right)\right\}\right] & \text { if } Y \text { is spectrally positive. }\end{cases}
$$

Thus, we insist that $\psi_{Y}$ be defined on $\mathbb{R}_{+}$, and we define its right inverse by

$$
\Phi_{Y}(q):=\sup \left\{\theta \geq 0: \psi_{Y}(\theta)=q\right\}, \quad q \geq 0 .
$$


We use also the notation

$$
\bar{Y}_{t}:=\sup _{0 \leq s \leq t} Y_{s} \quad \text { and } \quad \underline{Y}_{t}:=\inf _{0 \leq s \leq t} Y_{s}, \quad t \geq 0,
$$

and recall the duality lemma for Lévy processes (see, e.g. [2, p. 45]):

$$
\left\{Y_{t}-Y_{(t-s)-}: 0 \leq s \leq t\right\} \stackrel{\mathrm{D}}{=}\left\{Y_{s}: 0 \leq s \leq t\right\},
$$

where ' $\stackrel{\text { D }}{=}$ means equality in distribution. Hence,

$$
\sup _{0 \leq s \leq t}\left(Y_{t}-Y_{(t-s)-}\right) \stackrel{\mathrm{D}}{=} \bar{Y}_{t},
$$

which is equivalent to

$$
Y_{t}-\underline{Y}_{t} \stackrel{\mathrm{D}}{=} \bar{Y}_{t} .
$$

In this paper we shall mainly study the Lévy process $Y$ with the time parameter taking values in the whole of $\mathbb{R}$ (see Appendix B). The Skorokhod reflection mapping associated with $\left\{Y_{t}: t \in \mathbb{R}\right\}$ is defined (see Lemma 8 in Appendix A) via

$$
X_{t}:=\widetilde{\mathcal{R}}_{t} Y:=\sup _{-\infty<s \leq t}\left(Y_{t}-Y_{s}\right), \quad t \in \mathbb{R} .
$$

In the remaining part of this section we give conditions for the existence of the stationary process $\widetilde{\mathcal{R}} Y=\left(\widetilde{\mathcal{R}}_{t} Y, t \in \mathbb{R}\right)$, compute its marginal distribution, and define the local-time process $L$ of $\widetilde{R} Y$ at 0 which will be used for the construction of the fluid queue. (That this stationary process is Markov is easy to see due to the independence of the increments of $Y$.) A few words about the definition of $L$ are in order. We adopt the point of view that $L$ is a stationary random measure on $(\mathbb{R}, \mathscr{B})$, i.e.

$$
L(s, s+t]=L(0, t] \circ \theta_{s}, \quad t \geq 0, s \in \mathbb{R},
$$

where $\theta_{s}$ is the shift on the canonical space (see Appendix B) and $L$ regenerates together with $X$ at each point $t$ at which $X_{t}=0$. It is known (see, e.g. [4, pp. 61, 216] and [18, p. 144]) that $L$ is almost surely (a.s.) continuous if and only if the point $x=0$ is regular for the closed interval $(-\infty, 0]$ for the process $X$, and this is equivalent to $\inf \left\{t>0: Y_{t} \leq 0\right\}=0, \mathrm{P}_{0}$-a.s. Furthermore, $L$ is a.s. absolutely continuous if and only if, in addition to the above, the point $x=0$ is irregular for the open interval $(0, \infty)$ for the process $X$, and this is equivalent to $\inf \left\{t>0: Y_{t}>0\right\}>0, \mathrm{P}_{0}$-a.s. If $L$ is a.s. continuous then it is not difficult to attach a physical meaning to it as a cumulative input process to a secondary queue. For mathematical completeness, we shall also consider the case where $L$ is not a.s. continuous, in which case it can be shown to have a discrete support. In the continuous case, $L$ can be defined uniquely modulo a multiplicative constant. We shall make the normalisation precise later. In the discontinuous case, there is more freedom; however, insisting that its inverse be a subordinator, we are left with only one choice. The discontinuous case appears only once below and the construction of $L$ is discussed there. In all cases, the support of the measure $L$ is the closure of the set $\left\{t \in \mathbb{R}: X_{t}=0\right\}$.

Associated to the measure $(L(B), B \in \mathscr{B})$ we can define a cumulative local-time process, denoted (abusing notation) by the same letter and given by

$$
L_{t}:= \begin{cases}L(0, t], & t \geq 0 \\ -L(t, 0], & t<0 .\end{cases}
$$


The right-continuous inverse process is

$$
L_{x}^{-1}:= \begin{cases}\inf \{t>0: L(0, t]>x\}, & x \geq 0, \\ \sup \{t<0: L(t, 0]<x\}, & x<0 .\end{cases}
$$

In case $L$ is $\mathrm{P}$-a.s. continuous, the process $\left(L_{x}^{-1}, x \in \mathbb{R}\right)$ has, under $\mathrm{P}_{0}$, independent increments and a.s. increasing paths (i.e. it is a, possibly killed, subordinator). This is an additional requirement that needs to be imposed when $L$ is not P-a.s. continuous.

\subsection{Stationary reflection of a spectrally negative Lévy process}

Suppose that the process $Y$ is a spectrally negative Lévy process with nonmonotone paths; see (50), below.

Proposition 1. Let $Y=\left\{Y_{t}: t \in \mathbb{R}\right\}$ be a spectrally negative Lévy process with two-sided time. Assume that its Laplace exponent $\psi_{Y}(\theta)=\log \mathrm{E}\left[\exp \left\{\theta Y_{1}\right\}\right], \theta>0$, is such that $\psi_{Y}^{\prime}(0+)<0$. Then

$$
X=\left\{X_{t}:=\widetilde{\mathcal{R}}_{t} Y: t \in \mathbb{R}\right\}
$$

is the unique stationary solution of the Skorokhod dynamical system (SDS (see Appendix A)) driven by $Y$. The marginal distribution of $X$ is exponential with mean $1 / \Phi_{Y}(0)$.

Proof. Since $\mathrm{E}\left[Y_{t+1}-Y_{t}\right]=\psi_{Y}^{\prime}(0+)<0$, existence and uniqueness of the stationary solution is guaranteed by Corollary 2 in Appendix A. That $\Phi_{Y}(0)>0$ is a direct consequence of the definition of $\Phi_{Y}$ (see (3)). To derive the marginal distribution of $X$, consider, for $\beta \geq 0$,

$$
\mathrm{E}\left[\exp \left\{-\beta X_{0}\right\}\right]=\lim _{t \rightarrow \infty} \mathrm{E}_{0}\left[\exp \left\{-\beta\left(Y_{t}-\underline{Y}_{t}\right)\right\}\right]=\lim _{t \rightarrow \infty} \mathrm{E}_{0}\left[\exp \left\{-\beta \bar{Y}_{t}\right\}\right] .
$$

Since $Y$ is spectrally negative, its overall supremum, $\sup _{s \geq 0} Y_{s}$, is exponentially distributed with mean $1 / \Phi_{Y}(0)$ (see, e.g. [2, p. 190] or [18, p. 85]). This completes the proof.

In view of Proposition 1 we assume that

$$
\psi_{Y}^{\prime}(0+) \in[-\infty, 0)
$$

which is equivalent to

$$
\Phi_{Y}(0)>0 \text {. }
$$

It is easily seen that, for a nonmonotone spectrally negative $Y$, a necessary and sufficient condition for continuity of $L$ is that $Y$ has unbounded variation paths. This is further equivalent to $\sigma>0$ or $\int_{-1}^{0}|y| \Pi(\mathrm{d} y)=\infty$.

In the alternative case, when the paths of $Y$ are of bounded variation, the number of visits of $Y$ to its running infimum forms a discrete set. So

$$
n(s, t]:=\sum_{s<u \leq t} \mathbf{1}\left(X_{u}=0\right)
$$

is finite for all $-\infty<s<t<\infty$. Let $n_{t}:=n(0, t]$ if $t \geq 0$ and $n_{t}:=-n(t, 0]$ if $t<0$, and let $\left(\mathfrak{e}_{j}, j \in \mathbb{Z}\right)$ be a collection of independent and identically distributed exponentials with mean 1, independent of $Y$. We adopt the following construction for $L$ (which requires replacing the probability space $(\Omega, \mathcal{F}, \mathrm{P})$ by an obvious enlargement of it):

$$
L(s, t]=\sum_{n_{s}<i \leq n_{t}} \mathfrak{e}_{i}, \quad-\infty<s \leq t<\infty .
$$


In both cases, the process $\left(L_{x}^{-1}, x \in \mathbb{R}\right)$ is a subordinator under $\mathrm{P}_{0}$, with $L_{0}^{-1}=0, \mathrm{P}_{0}$-a.s. If $L$ is continuous, this property is immediate from the definition of $L$. If $L$ is discontinuous, $L^{-1}$ has independent increments due to our choice of the exponential jumps of $L$. Since $\psi_{Y}^{\prime}(0+)<0$, we have $Y_{t} \rightarrow \mp \infty$ as $t \rightarrow \pm \infty$, P-a.s., and this implies that $L_{t} \rightarrow \pm \infty$ as $t \rightarrow \pm \infty$, P-a.s. Thus, it is not possible for $L_{x}^{-1}$ to explode for finite $x$.

Regardless of the continuity of the paths of $L$, we always have the following proposition.

Proposition 2. Let $X$ and $Y$ be as in Proposition 1, and let $L$ be the local time at 0 of $X$. Then the local time $L$ can be normalised to satisfy

$$
\mathrm{E}_{0}\left[\exp \left\{-q L_{x}^{-1}\right\}\right]=\exp \left\{-\frac{x q}{\Phi_{Y}(q)}\right\}, \quad q \geq 0,
$$

and, moreover,

$$
\mathrm{E}_{0}\left[L_{x}^{-1}\right]=\frac{x}{\Phi_{Y}(0)}, \quad x \in \mathbb{R}, \quad \mathrm{E}\left[L_{t}\right]=t \Phi_{Y}(0), \quad t \in \mathbb{R} .
$$

Proof. For the Laplace exponent of $L_{x}^{-1}$ in (8), we refer the reader to [3, p. 731] (a result due to Fristedt [7]), [18], and [19]. The 'ladder process' $\left(\left(L_{x}^{-1}, X_{L_{x}^{-1}}\right), x \geq 0\right)$ is a Lévy process with values in $\mathbb{R}_{+}^{2}$ and Laplace exponent

$$
\hat{\kappa}(\alpha, \beta)=\log \mathrm{E}_{0}\left[\exp \left\{-\alpha L_{1}^{-1}-\beta X_{L_{1}^{-1}}\right\}\right]=\frac{\alpha-\psi_{Y}(\beta)}{\Phi_{Y}(\alpha)-\beta}
$$

obtained by Wiener-Hopf factorisation for a spectrally negative process; see [2, p. 191, Theorem 4]. Setting $\beta=0$ we obtain $\mathrm{E}_{0}\left[\exp \left\{-\alpha L_{1}^{-1}\right\}\right]=\exp \left\{-\alpha / \Phi_{Y}(\alpha)\right\}$, as claimed. From this we obtain $\mathrm{E}_{0}\left[L_{x}^{-1}\right]=x / \Phi_{Y}(0)$, by differentiation. Using the strong law of large numbers, we have $\lim _{x \rightarrow \infty} L_{x}^{-1} / x=1 / \Phi_{Y}(0), \mathrm{P}_{0}$-a.s., and, given that $\left(L_{x}^{-1}, x>0\right)$ is the right-continuous inverse function of $\left(L_{t}, t>0\right)$ (see (5)), we have $\lim _{t \rightarrow \infty} L_{t} / t=\Phi_{Y}(0), \mathrm{P}_{0}$-a.s., and P-a.s. Since $L$ is a stationary random measure, we have $\mathrm{E}\left[L_{t}\right]=C t$ for some constant $C$. Hence, we immediately have $C=\Phi_{Y}(0)$, and this proves (9).

We shall later need the P-distribution of the random variable

$$
D:=\inf \left\{t>0: X_{t}=0\right\} .
$$

Since $X_{0}$ is exponential with rate $\Phi_{Y}(0)$, we have $\mathrm{P}\left(X_{0}>0\right)=1$. So, if $0 \leq t<D$, we have $X_{t}=X_{0}+Y_{t}$, P-a.s. Therefore,

$$
D=\inf \left\{t>0: Y_{t}<-X_{0}\right\}, \quad \text { P-a.s. }
$$

Let

$$
\tau_{-x}:=\inf \left\{t>0: Y_{t}<-x\right\}
$$

Therefore,

$$
\begin{aligned}
\mathrm{E}\left[\mathrm{e}^{-\theta D}\right] & =\int_{0}^{\infty} \mathrm{P}\left(X_{0} \in \mathrm{d} x\right) \mathrm{E}\left[\exp \left\{-\theta \tau_{-x}\right\}\right] \\
& =\int_{0}^{\infty} \Phi_{Y}(0) \exp \left\{-\Phi_{Y}(0) x\right\}\left(Z^{(\theta)}(x)-\frac{\theta}{\Phi_{Y}(\theta)} W^{(\theta)}(x)\right) \mathrm{d} x \\
& =\Phi_{Y}(0) \frac{\psi_{Y}\left(\Phi_{Y}(0)\right) \Phi_{Y}(\theta)-\theta \Phi_{Y}(0)}{\Phi_{Y}(\theta) \Phi_{Y}(0)\left[\psi_{Y}\left(\Phi_{Y}(0)\right)-\theta\right]} \\
& =\frac{\Phi_{Y}(0)}{\Phi_{Y}(\theta)}
\end{aligned}
$$


where the first passage time formula, (55), given in Appendix B (see also [3, p. 732]), and the Laplace transforms for the scale functions $W^{(\theta)}$ and $Z^{(\theta)},(52)$ and (53), also given in Appendix B, have been used.

\subsection{Stationary reflection of a spectrally positive Lévy process}

We can repeat the construction in the subsection above for a spectrally positive Lévy process $Y$ with nonmonotone paths. We shall be using the formulae of Appendix B with $-Y$ in place of $Y$.

Proposition 3. Let $Y=\left\{Y_{t}: t \in \mathbb{R}\right\}$ be a spectrally positive Lévy process with two-sided time and Laplace exponent $\psi_{Y}$. Assume that its Laplace exponent $\psi_{Y}(\theta)=\log \mathrm{E}\left[\exp \left\{-\theta Y_{1}\right\}\right]$ is such that $\psi_{Y}^{\prime}(0+)>0$. Then the process $\left\{X_{t}:=\widetilde{\mathcal{R}}_{t} Y: t \in \mathbb{R}\right\}$ is the unique stationary solution to the SDS driven by $Y$. The stationary distribution of $X$ is given, for $\beta \geq 0$, by

$$
\mathrm{E}\left[\exp \left\{-\beta X_{0}\right\}\right]=\lim _{t \rightarrow \infty} \mathrm{E}_{0}\left[\exp \left\{-\beta\left(Y_{t}-\underline{Y}_{t}\right)\right\}\right]=\lim _{t \rightarrow \infty} \mathrm{E}_{0}\left[\exp \left\{-\beta \bar{Y}_{t}\right\}\right]=\psi_{Y}^{\prime}(0+) \frac{\beta}{\psi_{Y}(\beta)} .
$$

Proof. Note that in this case, by the assumption on $\psi_{Y}$,

$$
\mathrm{E}\left[Y_{t+1}-Y_{t}\right]=-\psi_{Y}^{\prime}(0+)<0,
$$

and, hence, $Y$ drifts to $-\infty$. Let $Z:=-Y$. Clearly, $Z$ is spectrally negative,

$$
X_{t}=Y_{t}-\underline{Y}_{t}=\bar{Z}_{t}-Z_{t}
$$

and $Z$ drifts to $+\infty$. The classical result due to Zolotarev [25] (see also [3, Proposition 5]) says that the stationary distribution of $X$ is as given in (11).

We shall therefore assume that

$$
\psi_{Y}^{\prime}(0+) \in(0, \infty)
$$

Hence, $\Phi_{Y}=\psi_{Y}^{-1}$ and so

$$
\Phi_{Y}^{\prime}(0+)=\frac{1}{\psi_{Y}^{\prime}(0+)} \in(0, \infty)
$$

It is easily seen that, starting from 0 , the process $Y$ hits $(-\infty, 0]$ immediately, P-a.s., and this ensures continuity of the local time $L$. Moreover, we may and do normalise $L$ so that

$$
L(s, t]=-\inf _{s<u \leq t} Y(s, u] .
$$

The continuity of $L$ implies that

$$
\left\{L_{x}^{-1}: x \geq 0\right\}=\left\{\tau_{-x}: x \geq 0\right\},
$$

where $\tau_{-x}:=\inf \left\{t>0: Y_{t}<-x\right\}=\inf \left\{t>0: Z_{t}>x\right\}$. Note that $\left(L_{x}^{-1}, x \in \mathbb{R}\right)$ is a subordinator under $\mathrm{P}_{0}$, with $L_{0}^{-1}=0, \mathrm{P}_{0}$-a.s. Furthermore, since $Y_{t}$ drifts to $\mp \infty$ as $t \rightarrow \pm \infty$, $L^{-1}$ is proper (not killed).

Let us briefly comment on the special case where, starting from $X_{0}=0$, the interval $(0, \infty)$ will be first visited by $X$ at an a.s. positive time. It is known [2, Chapter 7] that this occurs if and only if $Y$ has bounded variation, i.e.

$$
Y(s, t]=d_{Y}(t-s)+\int_{(s, t]} \int_{(0, \infty)} y \eta(\mathrm{d} u, \mathrm{~d} y),
$$


where $\eta$ is defined as a Poisson random measure on $\mathbb{R} \times \mathbb{R}_{+}$with intensity as in (49) of Appendix $\mathrm{B}, d_{Y}$ is the drift, and $\int_{0}^{1} y \Pi(\mathrm{d} y)<\infty$. Since we exclude the case where $Y$ is monotone, we must have $d_{Y}<0$. In this case, with (12) as the definition of $L$, it is known that, for all $s \leq t$,

$$
L(s, t]=\left|d_{Y}\right| \int_{s}^{t} \mathbf{1}\left(X_{u}=0\right) \mathrm{d} u .
$$

A rewording of the first part of Lemma 10 in Appendix B gives the first part of the following proposition.

Proposition 4. Let $Y$ be as in Proposition 3, and let $L$ be the local time at 0 of $X$. Then

$$
\mathrm{E}_{0}\left[\exp \left\{-q L_{x}^{-1}\right\}\right]=\exp \left\{-\Phi_{Y}(q) x\right\}, \quad q \geq 0 .
$$

Moreover,

$$
\mathrm{E}_{0}\left[L_{x}^{-1}\right]=x \Phi_{Y}^{\prime}(0+), \quad x \geq 0, \quad \mathrm{E}\left[L_{t}\right]=t \psi_{Y}^{\prime}(0+), \quad t \geq 0 .
$$

Proof. Equation (16) follows from (13) and the well-known characterisation of the distribution of the first hitting time $\tau_{x}^{+}$; see (54), below. By differentiating (16), we obtain the first part of (17), and using an ergodic argument (as in the proof of the second part of (9)), we obtain the second part of (17).

We now compute the P-distribution of $D=\inf \left\{t>0: X_{t}=0\right\}$ by arguing as earlier: we have $D=\inf \left\{t>0:-Y_{t}>X_{0}\right\}$, and, since $-Y$ is spectrally negative, we use the hitting time formula (54) in Appendix B to obtain

$$
\mathrm{E}\left[\mathrm{e}^{-\theta D}\right]=\mathrm{E}\left[\exp \left\{-\Phi_{Y}(\theta) X_{0}\right\}\right]=\psi_{Y}^{\prime}(0+) \frac{\Phi_{Y}(\theta)}{\psi_{Y}\left(\Phi_{Y}(\theta)\right)}=\psi_{Y}^{\prime}(0+) \frac{\Phi_{Y}(\theta)}{\theta},
$$

where we also used (11) and the fact that $\Phi_{Y} \circ \psi_{Y}$ is the identity function.

\section{Construction of (the stationary version of) the fluid queue with local-time input}

We wish to construct a fluid queue driven by

$$
\hat{L}(s, t]=L(s, t]-(t-s),
$$

where $L$ is the local time at 0 of the Markov process $X$. The process $X$ is a stationary Markov process which is the reflection of a spectrally negative (Subsection 2.1) or a spectrally positive (Subsection 2.2) Lévy process. In either case, $L$ is a stationary random measure with rate (see (9) and (17))

$$
\mu=\mathrm{E} L(s, s+1]= \begin{cases}\Phi_{Y}(0) & \text { if } Y \text { is spectrally negative, } \\ \psi_{Y}^{\prime}(0+) & \text { if } Y \text { is spectrally positive. }\end{cases}
$$

The fluid queue started from level $x$ at time 0 is, as explained in Appendix A, the process

$$
\left(\mathcal{R}_{0, t} \hat{L}(x), t \geq 0\right) \text {. }
$$

From Corollary 2 in Appendix A we immediately have the following theorem. 
Theorem 1. If $\mu<1$, there is a unique stationary version of the fluid queue driven by $\hat{L}$ and it is given by

$$
Q_{t}=\widetilde{\mathcal{R}}_{t} \hat{L}=\sup _{-\infty<u \leq t}\left(\hat{L}_{t}-\hat{L}_{u}\right), \quad t \in \mathbb{R} .
$$

Thus, the following assumptions will be made throughout the paper:

(A1) $\mu<1$,

(A2) if $Y$ is spectrally positive and of bounded variation then $d_{Y}<-1$.

Assumption (A1) arises so that we can construct a stationary version of $Q$ (as in Theorem 1). If $Y$ is spectrally positive, having nonmonotone paths of bounded variation, then its drift $d_{Y}$ must be negative. If, however, $\left|d_{Y}\right| \leq 1$ then (see (15)) $L(s, t] \leq t-s$ for all $s<t$ and so $Q$ will be identically equal to 0 .

Physically, we think of $Q$ as a stationary fluid queue whose cumulative input between times $s$ and $t$ is $L(s, t]$ and whose maximum potential output is $t-s$. Unlike $X$, the process $Q$ is not Markovian. However, since $Q$ has been built on the probability space supporting $X$, it makes sense to consider, for each $x \geq 0$, the probability measure $\mathrm{P}_{x}$ defined as $\mathrm{P}$ conditional on $\left\{X_{0}=x\right\}$.

Since $L$ is a stationary random measure with intensity $\mu$, we can define the Palm distribution with respect to it by

$$
\mathrm{P}_{L}(A)=\mu^{-1} \mathrm{E}\left[\int_{(0,1]} \mathbf{1}_{A} \circ \theta_{t} L(\mathrm{~d} t)\right], \quad A \in \mathcal{F} .
$$

We refer the reader to [9, Chapter 10] and [10, Chapter 11] for these notions. For a spectrally positive Lévy process $Y$, the random measure $L$ is continuous. For a spectrally negative Lévy process, $L$ is either continuous or discrete. Hence, $L$ is never a mixed measure.

If $L$ is a continuous random measure then it is known that $\mathrm{P}_{L}$ expresses conditioning with respect to the event that $t=0$ is a point of increase of $L$. But $L$ is the local time of $X$ at 0 . Therefore, the support of $L$ is the set of 0 s of $X$ and so $\mathrm{P}_{L}=\mathrm{P}_{0}$.

Now suppose that $L$ is a discrete random measure, as in (7). Let $n$ be the point process defined by (6) which is supported on the support of $L$. It is immediate that $\mathrm{P}_{0}$ is the Palm probability with respect to $n$. It can also be seen that $\mathrm{P}_{L}$ is related to $\mathrm{P}_{0}$ by 'size-biased averaging'.

Theorem 2. If $Y$ is a spectrally positive Lévy process or is spectrally negative with unbounded variation paths then the Palm probability $\mathrm{P}_{L}$ coincides with $\mathrm{P}_{0}$. If $Y$ is spectrally negative with bounded variation paths then (with L defined as in (7))

$$
\mathrm{P}_{L}(A)=\mathrm{E}_{0}\left[\mathfrak{e}_{0} \mathbf{1}_{A}\right],
$$

where $\mathfrak{e}_{0}$ is an independent rate-1 exponential.

This result follows straightforwardly from the Neveu exchange formula (see (26), below) between the Palm probabilities $\mathrm{P}_{L}$ and $\mathrm{P}_{0}$, and allows us to use the formulae of Appendix A involving Palm probabilities.

\section{Stationary distribution of the fluid queue}

We are interested in computing $\mathrm{P}\left(Q_{t} \in \cdot\right)$, a probability measure which is the same for all $t$. We will use three properties of $L$. First, duality, i.e. that $Y$ has the same distribution when 
time is reversed, see (4), implies that

$$
(L(0, t], t \geq 0) \stackrel{\mathrm{D}}{=}(L[-t, 0), t \geq 0) \quad \text { under } \mathrm{P} \text { and under } \mathrm{P}_{L} .
$$

Second, the process

$$
L_{x}^{-1}=\inf \{t \geq 0: L(0, t]>x\}, \quad x \geq 0
$$

is a subordinator under $\mathrm{P}_{0}$. Third, the Palm measure $\mathrm{P}_{L}$ coincides with $\mathrm{P}_{0}$.

Recall that $\psi_{Y}(\theta)$ has been defined as $\log \mathrm{E}\left[\exp \left\{\theta\left(Y_{t+1}-Y_{t}\right)\right\}\right]$ when $Y$ is spectrally negative and as $\log \mathrm{E}\left[\exp \left\{-\theta\left(Y_{t+1}-Y_{t}\right)\right\}\right]$ when $Y$ is spectrally positive. The reason is that it is customary and convenient to have $\theta \geq 0$ in both cases. Recall also that $\Phi_{Y}(q)=\sup \left\{\theta \geq 0: \psi_{Y}(\theta)=q\right\}$. The stationary distribution of $Q$ will be expressed in terms of $\psi_{Y}$. For earlier works on this problem, we refer the reader to [22] for diffusion local times and [23] for the inverse of a general subordinator. The present formulation in Theorem 3, below, is in particular tailored for the local time of $X$. The proof method, making use of Palm probabilities, is new.

First, since we allow discontinuous local times, the following simple lemma is needed. We omit the proof.

Lemma 1. Let $\overline{\mathbb{R}}:=\mathbb{R} \cup\{+\infty,-\infty\}$. If $h: \overline{\mathbb{R}} \rightarrow \overline{\mathbb{R}}$ is right continuous and nondecreasing then $h^{-1}(x):=\inf \{t: h(t)>x\}, x \in \mathbb{R}$, is right continuous and nondecreasing, $h^{-1}: \overline{\mathbb{R}} \rightarrow \overline{\mathbb{R}}$, and, for all $t \in \overline{\mathbb{R}}$,

$$
h^{-1}(h(t-)-) \leq h^{-1}(h(t)-) \leq t \leq h^{-1}(h(t-)) \leq h^{-1}(h(t)) .
$$

Furthermore, $\left(h^{-1}\right)^{-1}=h$.

Theorem 3. (i) If $X$ is the reflection of a spectrally negative Lévy process $Y$ with $\psi_{Y}^{\prime}(0+)<0$ and $\psi_{Y}(1)>0$ then

$$
\mathrm{P}_{0}\left(Q_{0}>a\right)=\exp \left\{-\psi_{Y}(1) a\right\}, \quad \mathrm{P}\left(Q_{0}>a\right)=\Phi_{Y}(0) \exp \left\{-\psi_{Y}(1) a\right\}, \quad a \geq 0 .
$$

(ii) If $X$ is the reflection of a spectrally positive Lévy process $Y$ with $0<\psi_{Y}^{\prime}(0+)<1$, and $d_{Y}<-1$ in the case of bounded variation, then

$$
\mathrm{P}_{0}\left(Q_{0}>a\right)=\mathrm{e}^{-\theta^{*} a}, \quad \mathrm{P}\left(Q_{0}>a\right)=\psi_{Y}^{\prime}(0+) \mathrm{e}^{-\theta^{*} a}, \quad a \geq 0,
$$

where $\theta^{*}>0$ is defined by $\psi_{Y}\left(\theta^{*}\right)=\theta^{*}$.

Proof. By the construction of $Q$ and duality, we have

$$
\mathrm{P}\left(Q_{0} \leq a\right)=\mathrm{P}\left(\sup _{u \geq 0}\left(L_{u}-u\right) \leq a\right) .
$$

Note that our assumptions imply that the process $\left\{L_{t}-t: t \geq 0\right\}$ does not have monotone paths; nevertheless, the event $\left\{\sup _{\{t \geq 0\}}\left(L_{t}-t\right) \leq a\right\}$ can be expressed in terms of $L^{-1}$ :

$$
\left\{\sup _{t \geq 0}\left(L_{t}-t\right) \leq a\right\}=\left\{\sup _{x \geq 0}\left(x-L_{x}^{-1}\right) \leq a\right\} .
$$

To justify this (recall that in case (i) $L$ is not necessarily continuous), we first assume that $L_{t} \leq t+a$ for all $t \geq 0$. Hence, $L_{L_{x}^{-1}} \leq L_{x}^{-1}+a$ for all $x \geq 0$. Since (Lemma 1 )

$$
L_{L_{x}^{-1}} \geq x, \quad x \geq 0,
$$


we have $x \leq L_{x}^{-1}+a$ for all $x \geq 0$ and, thus, $\sup _{x \geq 0}\left(x-L_{x}^{-1}\right) \leq a$. Next assume that $x-L_{x}^{-1} \leq \bar{a}$ for all $x \geq 0$. Then $L_{x-\varepsilon}^{-1} \geq x-\varepsilon-a$ for all $x \geq \varepsilon>0$. Letting $\varepsilon \downarrow 0$ we obtain $L_{x-}^{-1} \geq x-a$ for all $x \geq 0$. Therefore, $L_{\left(L_{t}\right)-}^{-1} \geq L_{t}-a$ for all $t \geq 0$. Since (by Lemma 1 again)

$$
L_{\left(L_{t}\right)-}^{-1} \leq t, \quad t \geq 0
$$

we obtain $L_{\left(L_{t}\right)-}^{-1} \geq t-a$ for all $t \geq 0$ and this gives $\sup _{t \geq 0}\left(L_{t}-t\right) \leq a$. We first compute the $\mathrm{P}_{0}$-distribution of $Q_{0}$ :

$$
\mathrm{P}_{0}\left(Q_{0}>a\right)=\mathrm{P}_{0}\left(\sup _{x \geq 0}\left(x-L_{x}^{-1}\right)>a\right), \quad a \geq 0 .
$$

Under $\mathrm{P}_{0}$, the process

$$
\left\{\Lambda_{x}:=x-L_{x}^{-1}: x \geq 0\right\}
$$

is a spectrally negative Lévy process with bounded variation paths. Letting

$$
\sigma_{a}:=\inf \left\{x: \Lambda_{x}>a\right\},
$$

and applying Lemma 10 in Appendix B, we have

$$
\begin{aligned}
\mathrm{P}_{0}\left(Q_{0}>a\right) & =\mathrm{P}_{0}\left(\sigma_{a}<\infty\right) \\
& =\lim _{q \downarrow 0} \mathrm{E}_{0}\left[\exp \left\{-q \sigma_{a}\right\}\right] \\
& =\lim _{q \downarrow 0} \exp \left\{-\Phi_{\Lambda}(q) a\right\} \\
& =\exp \left\{-\Phi_{\Lambda}(0) a\right\} .
\end{aligned}
$$

The function $\Phi_{\Lambda}(q)$ is given by

$$
\Phi_{\Lambda}(q)=\sup \left\{\theta \geq 0: \psi_{\Lambda}(\theta)=q\right\}, \quad q \geq 0
$$

(see (51)), where

$$
\psi_{\Lambda}(\theta)=\log \mathrm{E}_{0}\left[\exp \left\{\theta \Lambda_{1}\right\}\right]=\theta+\log \mathrm{E}_{0}\left[\exp \left\{-\theta L_{1}^{-1}\right\}\right] .
$$

If $Y$ is spectrally negative then we use Proposition 2 for an expression for $\log \mathrm{E}_{0}\left[\exp \left\{-\theta L_{1}^{-1}\right\}\right]$. If $Y$ is spectrally positive, we use Proposition 4. We obtain

$$
\psi_{\Lambda}(\theta)= \begin{cases}\theta-\frac{\theta}{\Phi_{Y}(\theta)} & \text { if } Y \text { is spectrally negative } \\ \theta-\Phi_{Y}(\theta) & \text { if } Y \text { is spectrally positive. }\end{cases}
$$

In both cases, $Q_{0}$ is exponential under $\mathrm{P}_{0}$ with parameter $\Phi_{\Lambda}(0)$, which has a different value in each case.

Let $\mu$ be the rate of $L$ (see (19)). Using (48), below, we have, for any measurable function $g: \mathbb{R}_{+} \rightarrow \mathbb{R}_{+}$

$$
\mathrm{E}\left[g\left(Q_{0}\right)\right]=(1-\mu) g(0)+\mu \mathrm{E}_{L}\left[\frac{\int_{Q_{0-}}^{Q_{0}} g(x) \mathrm{d} x}{Q_{0}-Q_{0-}}\right],
$$

where the last fraction is defined to be $g\left(Q_{0}\right)$ on the event $\left\{Q_{0}=Q_{0-}\right\}$. 
First suppose that $Y$ is spectrally negative with unbounded variation paths or spectrally positive. Then $L$ is continuous. Therefore, $Q$ has continuous sample paths, so $Q_{0}=Q_{0-}$ a.s. By Theorem 2, we have $\mathrm{P}_{L}=\mathrm{P}_{0}$. Therefore,

$$
\mathrm{E}\left[g\left(Q_{0}\right)\right]=(1-\mu) g(0)+\mu \mathrm{E}_{0}\left[g\left(Q_{0}\right)\right] .
$$

Let $a>0$, and consider the function $g(x):=\mathbf{1}(x>a), x \geq 0$. We then have

$$
\mathrm{P}\left(Q_{0}>a\right)=\mu \mathrm{P}_{0}\left(Q_{0}>a\right)=\mu \exp \left\{-\Phi_{\Lambda}(0) a\right\} .
$$

Next suppose that $Y$ is spectrally negative with bounded variation paths. Then $L$ is discrete, given by (7). In this case,

$$
Q_{0}=Q_{0-}+\mathfrak{e}_{0}, \quad \mathrm{P}_{0} \text {-a.s., }
$$

where $\mathfrak{e}_{0}$ is rate- 1 exponential and independent of $Q_{0-}$ both under $\mathrm{P}$ and $\mathrm{P}_{0}$. Therefore,

$$
\mathrm{E}_{0}\left[\exp \left\{-\theta Q_{0}\right\}\right]=\mathrm{E}_{0}\left[\exp \left\{-\theta Q_{0-}\right\}\right] \mathrm{E}_{0}\left[\exp \left\{-\theta \mathfrak{e}_{0}\right\}\right] .
$$

But $\mathrm{E}_{0}\left[\exp \left\{-\theta \mathfrak{e}_{0}\right\}\right]=1 /(\theta+1)$ and $\mathrm{E}_{0}\left[\exp \left\{-\theta Q_{0}\right\}\right]=\Phi_{\Lambda}(0) /\left(\theta+\Phi_{\Lambda}(0)\right)$. So

$$
\mathrm{E}_{0}\left[\exp \left\{-\theta Q_{0-}\right\}\right]=\frac{(\theta+1) \Phi_{\Lambda}(0)}{\theta+\Phi_{\Lambda}(0)}
$$

Now consider (22). Here, $Q_{0}-Q_{0-}=\mathfrak{e}_{0}>0, \mathrm{P}_{L}$-a.s. Also, by Theorem $2, \mathrm{E}_{L}[Z]=\mathrm{E}_{0}\left[Z \mathfrak{e}_{0}\right]$ for any (say, positive) random variable $Z$. Hence,

$$
\mathrm{E}\left[g\left(Q_{0}\right)\right]=(1-\mu) g(0)+\mu \mathrm{E}_{0}\left[\int_{Q_{0-}}^{Q_{0-}+\mathfrak{e}_{0}} g(x) \mathrm{d} x\right] .
$$

Apply this with the function $g(x):=\mathrm{e}^{-\theta x}$ :

$$
\begin{aligned}
\mathrm{E}\left[\exp \left\{-\theta Q_{0}\right\}\right] & =(1-\mu)+\frac{\mu}{\theta} \mathrm{E}_{0}\left[\exp \left\{-\theta Q_{0}-\right\}\left(1-\exp \left\{-\theta \mathfrak{e}_{0}\right\}\right)\right] \\
& =(1-\mu)+\frac{\mu}{\theta} \mathrm{E}_{0}\left[\exp \left\{-\theta Q_{0}-\right\}\right] \mathrm{E}_{0}\left[1-\exp \left\{-\theta \mathfrak{e}_{0}\right\}\right] \\
& =(1-\mu)+\frac{\mu}{\theta} \frac{(\theta+1) \Phi_{\Lambda}(0)}{\theta+\Phi_{\Lambda}(0)}\left(1-\frac{1}{\theta+1}\right) \\
& =(1-\mu)+\mu \frac{\Phi_{\Lambda}(0)}{\theta+\Phi_{\Lambda}(0)}
\end{aligned}
$$

which again implies that (23) holds.

The proof will be complete once we show that

$$
\Phi_{\Lambda}(0)= \begin{cases}\psi_{Y}(1) & \text { if } Y \text { is spectrally negative } \\ \theta^{*} & \text { if } Y \text { is spectrally positive }\end{cases}
$$

Note that $\Phi_{\Lambda}(0)$ is the positive solution of $\psi_{\Lambda}(\theta)=0$. If $Y$ is spectrally negative, we see from (21) that $\psi_{\Lambda}(\theta)=0$ if and only if $\Phi_{Y}(\theta)=1$ and, by the definition of $\Phi_{Y}$, the latter is true if and only if $\theta=\psi_{Y}(1)$. Thus, $\Phi_{\Lambda}(0)=\psi_{Y}(1)$. If $Y$ is spectrally positive, $\psi_{\Lambda}(\theta)=0$ if and only if $\Phi_{Y}(\theta)=\theta$ if and only if $\theta=\psi_{Y}(\theta)$. See Table 1 . 
TABLE 1: Table showing the basic characteristics of the system in both cases.

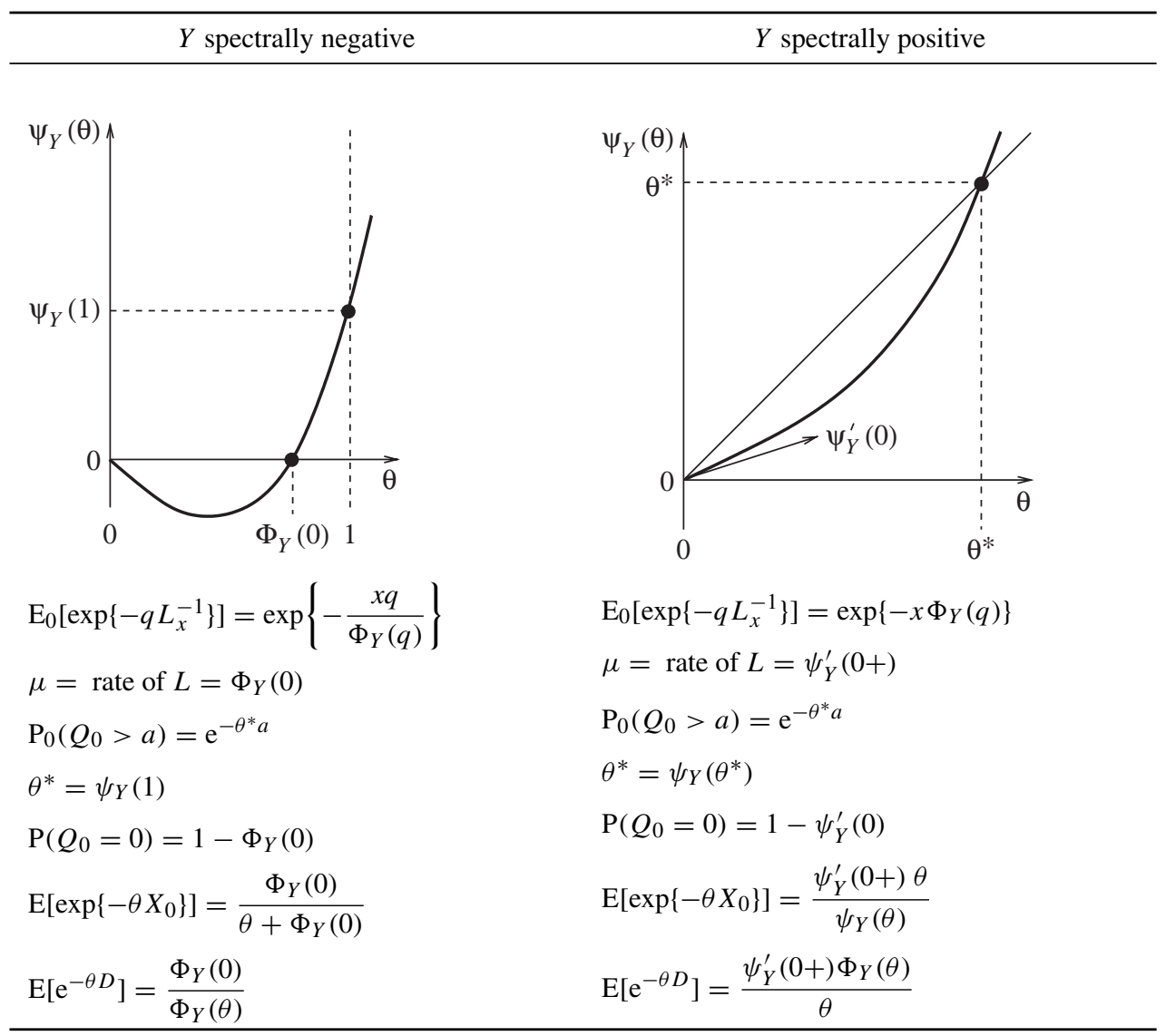

\subsection{Example 1: fluid queue driven by the local time of a reflected Brownian motion}

Consider $Y$ to be a Brownian motion with drift (see also [17], [20], and [22]):

$$
Y_{t}:=\sigma B_{t}-\mu t, \quad t \in \mathbb{R},
$$

where $\sigma>0$ and $\mu>0$. Here $B=\left(B_{t}, t \in \mathbb{R}\right)$ is a standard Brownian motion with twosided time. In other words, $\left(B_{t}, t \geq 0\right)$ and $\left(B_{-t}, t \geq 0\right)$ are independent standard Brownian motions with $B_{0}=0$ (although specification of $B_{0}$ does not affect the results below). The Lévy measure here is 0 . Consider $Y$ as in Subsection 2.2, and let

$$
\psi_{Y}(\theta)=\log \mathrm{E}\left[\exp \left\{-\theta Y_{1}\right\}\right]=\frac{1}{2} \sigma^{2} \theta^{2}+\mu \theta, \quad \theta>0 .
$$

Define

$$
X_{t}=\widetilde{\mathcal{R}}_{t} Y=\sup _{-\infty<s \leq t}\left(\sigma\left(B_{t}-B_{s}\right)-\mu(t-s)\right), \quad t \in \mathbb{R} .
$$

Lemma 3, below, gives the distribution of $X_{0}$ under P (see also [5, p. 129]):

$$
\mathrm{E}\left[\exp \left\{-\beta X_{0}\right\}\right]=\psi_{Y}^{\prime}(0+) \frac{\beta}{\psi_{Y}(\beta)}=\frac{\mu}{\sigma^{2} \beta / 2+\mu},
$$


i.e. exponential with rate $2 \mu / \sigma^{2}$. Let $L$ be the local time at 0 of $X$. The rate of $L$ (see (19)) is $\psi_{Y}^{\prime}(0+)=\mu$. Assume that $\mu<1$. Let $\hat{L}(s, t]=L(s, t]-(t-s)$, and let $Q$ be defined by

$$
Q_{t}=\widetilde{\mathcal{R}}_{t} \hat{L}, \quad t \in \mathbb{R} .
$$

Theorem 3 gives the distribution of $Q_{0}$ under P:

$$
\mathrm{P}\left(Q_{0}>a\right)=\psi_{Y}^{\prime}(0+) \mathrm{e}^{-\theta^{*} a}=\mu \exp \left\{-\frac{2(1-\mu) a}{\sigma^{2}}\right\}, \quad a \geq 0 .
$$

Here, $\theta^{*}$ was found from $\psi_{Y}\left(\theta^{*}\right)=\theta^{*}$. Thus, $Q_{0}$ is a mixture of an exponential with rate $2(1-\mu) / \sigma^{2}$ and the constant 0 , which is assumed with probability $\mu$.

\subsection{Example 2: fluid queue driven by the local time of a compound Poisson process with drift}

Suppose that, for $\alpha>0$,

$$
Y_{t}=S_{t}-\alpha t, \quad t \in \mathbb{R},
$$

where $S$ is a compound Poisson process with only positive jumps, jump rate $\lambda$, and jump size distribution $F$. For simplicity, we take $F$ to be exponential with rate $\delta>0$, i.e. $F(\mathrm{~d} x)=$ $\delta \mathrm{e}^{-\delta x} \mathrm{~d} x$. Then

$$
\begin{aligned}
\psi_{Y}(\theta) & =\log \mathrm{E}\left[\exp \left\{-\theta\left(Y_{t+1}-Y_{t}\right)\right\}\right] \\
& =\alpha \theta-\lambda \int_{[0, \infty)}\left(1-\mathrm{e}^{-\theta x}\right) F(\mathrm{~d} x) \\
& =\alpha \theta-\frac{\lambda \theta}{\delta+\theta}, \quad \theta>0
\end{aligned}
$$

The assumption $0<\psi^{\prime}(0+)<1$ implies that $1+\lambda / \delta>\alpha>\lambda / \delta$. Moreover, the assumption $\left|d_{Y}\right|>1$ additionally implies that $\alpha>1$. We can define the background stationary Markov process by

$$
X_{t}=\widetilde{R}_{t} Y=\sup _{-\infty<s \leq t}\left(S_{t}-S_{s}-\alpha(t-s)\right), \quad t \in \mathbb{R} .
$$

We have

$$
\mathrm{E}\left[\exp \left\{-\beta X_{0}\right\}\right]=\frac{\alpha-\lambda m}{\alpha-\lambda \int_{[0, \infty)}\left(\left(1-\mathrm{e}^{-\beta x}\right) / \beta\right) F(\mathrm{~d} x)} .
$$

Unlike the previous example, here $\mathrm{P}\left(X_{0}=0\right)=\lim _{\beta \uparrow \infty} \mathrm{E}\left[\exp \left\{-\beta X_{0}\right\}\right]=\alpha-\lambda / \delta$ is positive. The local time $L$ of $X$ at 0 has rate (cf. (17))

$$
\mu=\psi_{Y}^{\prime}(0+)=\alpha-\frac{\lambda}{\delta} .
$$

The assumptions on $\alpha$ imply that $\mu<1$ and, hence, we can construct the stationary process $Q$ by $Q_{t}=\widetilde{\mathcal{R}}_{t} \hat{L}$, where $\hat{L}(s, t]=L(s, t]-(t-s)$. We have

$$
\mathrm{P}\left(Q_{0}>x\right)=\mu \mathrm{e}^{-\theta^{*} x},
$$

where $\theta^{*}=\psi_{Y}\left(\theta^{*}\right)=\lambda(\alpha-1)^{-1}-\delta$. Note that the latter is positive since $\alpha>1$ and $1+\lambda / \delta>\alpha$. 


\subsection{Example 3: fluid queue driven by the local time of a risk-type process}

Let

$$
Y_{t}=b t-S_{t}, \quad t \in \mathbb{R},
$$

where $b>0, S$ is an $\alpha$-stable subordinator, $0<\alpha<1$, with

$$
\mathrm{E}\left[\exp \left\{-\theta\left(S_{t+1}-S_{t}\right)\right\}\right]=\exp \left\{-c \theta^{\alpha}\right\}, \quad \theta>0,
$$

and $c$ is a positive constant. Thus, $S$ is a $(1 / \alpha)$-self-similar process, i.e. $\left(S_{\kappa t}, t \in \mathbb{R}\right) \stackrel{\mathrm{D}}{=}\left(\kappa^{1 / \alpha} S_{t}\right.$, $t \in \mathbb{R})$. We here have $\mathrm{E}\left[S_{t}\right]=+\infty$ for $t>0$ and $S_{t} \rightarrow \infty$ faster than linearly, so $Y_{t} \rightarrow-\infty$ as $t \rightarrow \infty$ a.s. Similarly, $S_{t} \rightarrow-\infty$ as $t \rightarrow-\infty$ a.s. So the stationary reflection of $Y$,

$$
X_{t}=\widetilde{\mathcal{R}}_{t} Y=\sup _{-\infty<s \leq t}\left(b(t-s)-\left(S_{t}-S_{s}\right)\right), \quad t \in \mathbb{R},
$$

exists uniquely, due to Lemma 8 in Appendix A. Physically, $X_{t}$ is the content of a queue with linear input (arriving at rate $b$ ) and jump-type service represented by $S$. Alternatively, $X$ is a so-called risk process in the theory of risk. We have

$$
\psi_{Y}(\theta)=\log \mathrm{E}\left[\exp \left\{-\theta\left(Y_{t+1}-Y_{t}\right)\right\}\right]=b \theta-c \theta^{\alpha}, \quad \theta \geq 0 .
$$

We refer the reader to Subsection 2.1 and, specifically, Lemma 1, for the distribution of $X_{0}$, which is exponential with rate $\mu>0$, where $\mu$ satisfies $\psi_{Y}(\mu)=0$, i.e.

$$
\mu=\left(\frac{c}{b}\right)^{1 /(1-\alpha)}
$$

The local time $L$ of $X$ at 0 is such that $t \mapsto L(0, t]$ is a.s. right continuous (but not continuous) with rate $\mu$. Assuming that $\mu<1$, or $c<b$, we can further let $\hat{L}(s, t]=L(s, t]-(t-s)$ and let $Q$ be defined by

$$
Q_{t}=\widetilde{R}_{t} \hat{L}, \quad t \in \mathbb{R}
$$

(see Lemma 1). Theorem 3 gives the distribution of $Q_{0}$ under $\mathrm{P}_{0}$ and under $\mathrm{P}$. We have

$$
\mathrm{P}_{0}\left(Q_{0}>x\right)=\mathrm{e}^{-(b-c) x}, \quad \mathrm{P}\left(Q_{0}>x\right)=\left(\frac{c}{b}\right)^{1 /(1-\alpha)} \mathrm{e}^{-(b-c) x}, \quad x \geq 0 .
$$

\subsection{Example 4: fluid queue driven by the local time of a risk-type process with a Brownian component}

Take

$$
Y_{t}=3 b t+\sigma B_{t}-S_{t},
$$

where $S$ is the inverse local time of an independent Brownian motion, i.e. a $\frac{1}{2}$-stable subordinator. Assume that $\sigma^{2}>0$. We have

$$
\psi_{Y}(\theta)=\log \mathrm{E}\left[\exp \left\{\theta\left(Y_{1}-Y_{0}\right)\right\}\right]=3 b \theta+\frac{1}{2} \sigma^{2} \theta^{2}-2 c \theta^{1 / 2}, \quad \theta>0,
$$

where $c$ is a scaling parameter. Since $\lim _{t \rightarrow \infty} Y_{t}=-\infty$ a.s., Lemma 8 in Appendix A allows us to construct $X_{t}=\widetilde{\mathcal{R}}_{t} Y$. Here, $Y$ is spectrally negative, and so, as shown in Lemma 1 ,

$$
\mathrm{P}\left(X_{0}>x\right)=\mathrm{e}^{-\mu x}, \quad x>0,
$$


where $\mu>0$ and

$$
\psi_{Y}(\mu)=0
$$

Letting

$$
\delta=1+b^{3} \sigma^{-2} c^{-2}
$$

we find that

$$
\mu=2\left(\frac{c}{\sigma^{2}}\right)^{2 / 3} \frac{\left(\delta^{7}+1\right)^{1 / 3}+\left(\delta^{7}-1\right)^{1 / 3}}{\delta^{2}} .
$$

Here, $\mathrm{P}\left(X_{0}=0\right)=0$. As in Proposition 2, this $\mu$ is the rate of the local time $L$ of $X$. Note that, since $Y$ has unbounded variation paths, the local time $L$ is a.s. continuous. Assuming that $\mu<1$, which is equivalent to

$$
\psi_{Y}(1)=b+\frac{1}{2} \sigma^{2}-c>0,
$$

we construct $Q$ as before: $Q_{t}=\widetilde{\mathcal{R}}_{t} \hat{L}, t \in \mathbb{R}$. From Theorem 3 we have

$$
\mathrm{P}\left(Q_{0}>x\right)=\mu \exp \left\{-\left(b+\frac{1}{2} \sigma^{2}-c\right) x\right\}, \quad x>0 .
$$

\section{Idle and busy periods}

In this section we study idle and busy periods of the fluid queue process $\left\{Q_{t}: t \in \mathbb{R}\right\}$, as defined in (20). We work under the assumptions that $Y$ is either spectrally negative or spectrally positive and that $0<\mu<1$, where $\mu$ is the rate of $L$; see (19). Under these assumptions, the interior of the set $\left\{t \in \mathbb{R}: Q_{t}=0\right\}$ is the union of disjoint open intervals $(g(n), d(n)), n \in \mathbb{Z}$. We need a convention for the enumeration of the endpoints of these intervals, and here is the one we adopt.

First,

$$
\cdots<g(-1)<g(0) \leq 0<g(1)<g(2)<\cdots .
$$

Second,

$$
g(n)<d(n)<g(n+1), \quad n \in \mathbb{Z} .
$$

Figures 1, 2, and 3 exemplify how one should visualize idle and busy periods of sample paths of $Q$ for three different examples of driving Lévy processes. Let $N_{1}$ and $N_{2}$ be the random measures (point processes) that put mass 1 to each of the points $g(n)$ and $d(n)$, respectively. Note that the point processes $N_{1}$ and $N_{2}$ are jointly stationary.

The intervals $(g(n), d(n))$ are called idle periods, while the intervals $(d(n), g(n+1))$ are called busy periods. An observed idle period is, by definition, equal in distribution to an idle period, given that the idle period contains a fixed time $t$ of observation. By stationarity, we may take the time of observation to be $t=0$. In other words,

observed idle period $:=\left((g(0), d(0)) \mid Q_{0}=0\right) \stackrel{\mathrm{D}}{=}((g(0), d(0)) \mid g(0)<0<d(0))$.

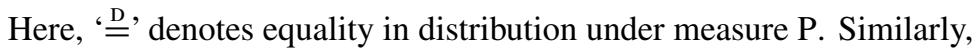

$$
\text { observed busy period :=((d(0), } \left.g(1)) \mid Q_{0}>0\right) \stackrel{\mathrm{D}}{=}((d(0), g(1)) \mid d(0)<0<g(1)) .
$$

In this section we identify the distribution of observed idle (and busy) periods (see Propositions 5 and 6, below). In both cases we shall appeal to the result of Lemma 2, below, a short proof of which is provided. Note that (25), below, and related facts are also proved in [17, Section 5] and [23]. 
Lemma 2. Let $(\Omega, \mathcal{F}, \mathrm{P})$ be a probability space endowed with a $\mathrm{P}$-preserving flow $\left(\theta_{t}, t \in \mathbb{R}\right)$ (see Appendix $A$ ). Let $N_{1}$ and $N_{2}$ be jointly stationary simple random point processes $\left(N_{i} \circ \theta_{t}(B)=N_{i}(B+t), t \in \mathbb{R}, B \in \mathcal{B}(\mathbb{R}), i=1,2\right)$ with points $\left\{t_{i}(n), n \in \mathbb{Z}\right\}, i=1,2$, such that

$$
\cdots<t_{1}(-1)<t_{1}(0) \leq 0<t_{1}(1)<t_{1}(2)<\cdots
$$

and

$$
t_{1}(n)<t_{2}(n)<t_{1}(n+1) \text { for all } n \in \mathbb{Z} \text {. }
$$

Let $M$ be the random measure which is defined through its derivative with respect to the Lebesgue measure as

$$
\frac{M(\mathrm{~d} t)}{\mathrm{d} t}=\sum_{n \in \mathbb{Z}} \mathbf{1}\left(t_{1}(n)<t<t_{2}(n)\right) .
$$

Assume that $N_{1}$ has finite intensity. Let $\mathrm{P}_{M}$ be the Palm probability with respect to $M$. Then

$$
\mathrm{E}_{M}\left[\exp \left\{-\alpha t_{2}(0)+\beta t_{1}(0)\right\}\right]=\frac{\alpha \mathrm{E}_{M}\left[\exp \left\{-\alpha t_{2}(0)\right\}\right]-\beta \mathrm{E}_{M}\left[\exp \left\{-\beta t_{2}(0)\right\}\right]}{\alpha-\beta}, \quad \alpha, \beta>0 .
$$

Proof. It is easy to see that $M$ is also stationary, i.e. $M \circ \theta_{t}(B)=M(B+t)$. Let $\mathrm{P}_{N_{i}}$ be the Palm probability with respect to $N_{i}, i=1,2$, and let $\lambda$ be the intensity of $N_{1}$ (which is, due to the law of large numbers, the same as the intensity of $N_{2}$ ). It follows easily from Campbell's formula that $M$ has finite intensity: $\lambda_{M}=\lambda \mathrm{E}_{N_{1}}\left[t_{2}(0)-t_{1}(0)\right]<\infty$. The Palm exchange formula between $\mathrm{P}_{M}$ and $\mathrm{P}_{N_{1}}$ yields

$$
\lambda_{M} \mathrm{E}_{M}[Y]=\lambda \mathrm{E}_{N_{1}}\left[\int_{t_{1}(0)}^{t_{1}(1)} Y \circ \theta_{t} M(\mathrm{~d} t)\right]=\lambda \mathrm{E}_{N_{1}}\left[\int_{t_{1}(0)}^{t_{2}(0)} Y \circ \theta_{t} \mathrm{~d} t\right]
$$

for any bounded random variable $Y$. (In [1, p. 21], the formula is given and proved for point processes, but the generalisation for arbitrary jointly stationary random measures is

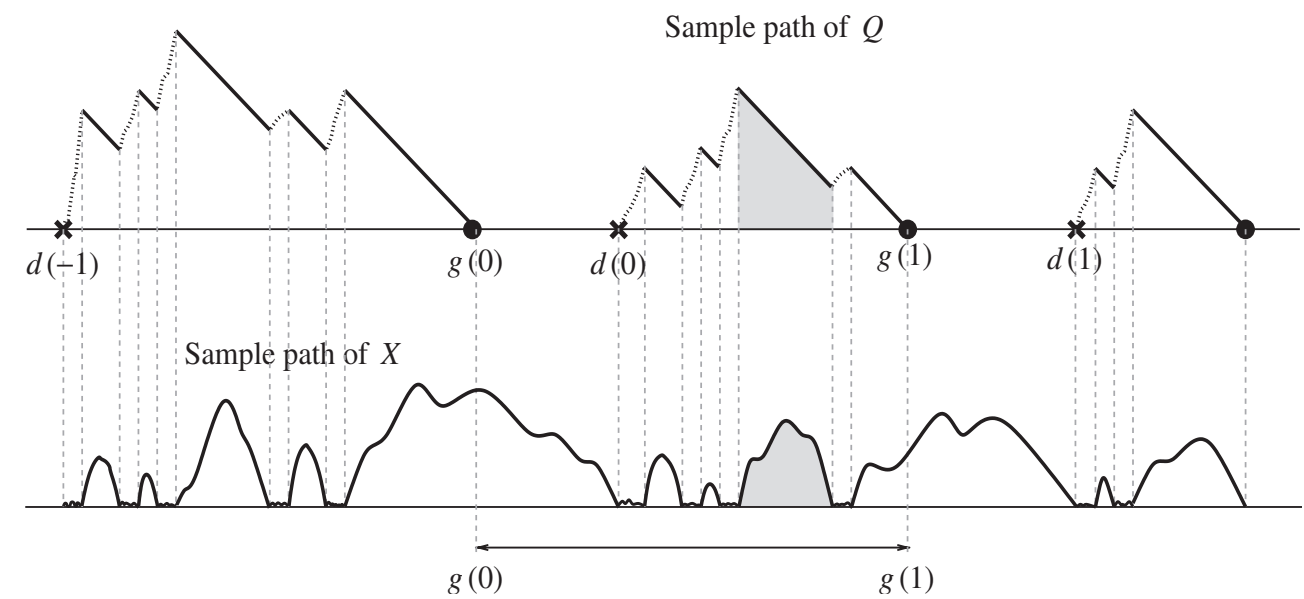

Figure 1: Typical behaviour of $Q$ and the background Markov process $X$ when the underlying Lévy process $Y$ has unbounded variation paths. By convention, the origin of time is contained between $g(0)$ and $d(0)$. Note that excursions of $X$ away from 0 correspond to intervals over which $Q$ decreases. 


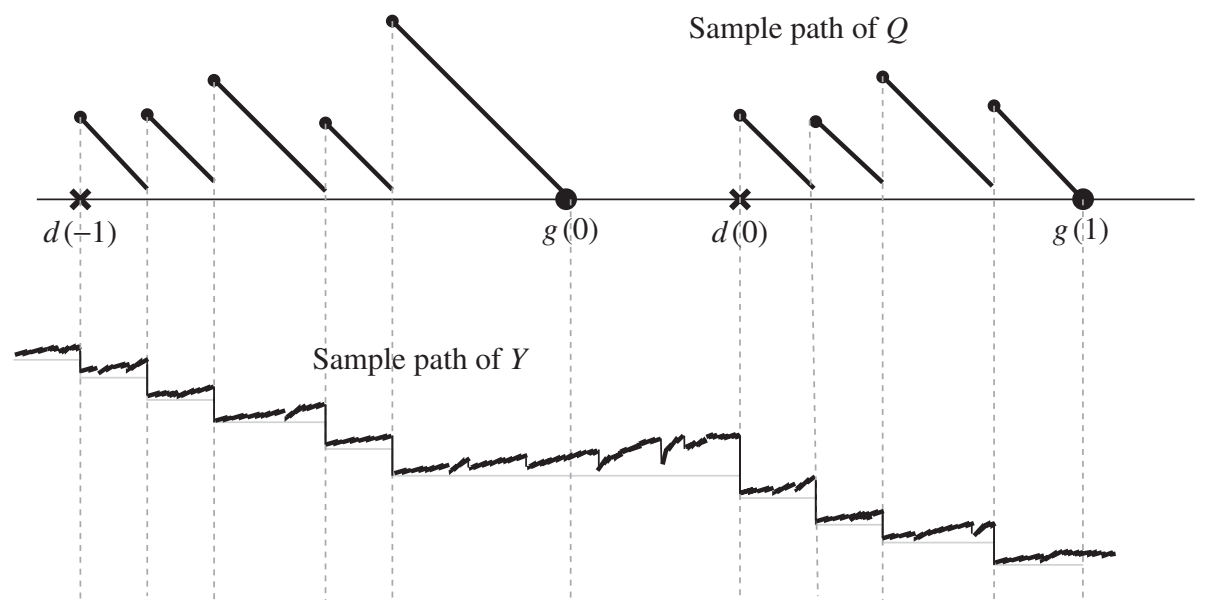

FIGURE 2: Typical behaviour of $Q$ and the background Lévy process $Y$ for the case in which $Y$ is spectrally negative with bounded variation paths.

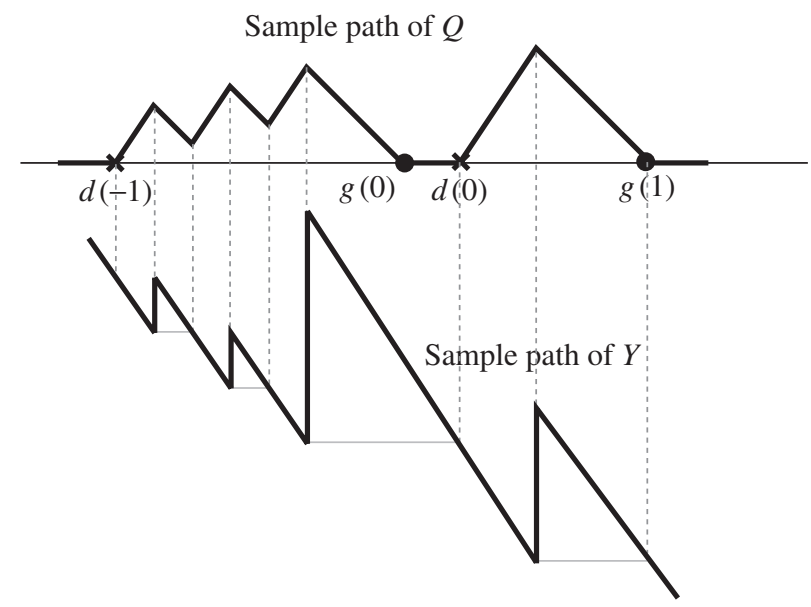

Figure 3: Typical behaviour of $Q$ and the background Lévy process $Y$ for the case in which $Y$ is spectrally positive with bounded variation paths. Here, only the case where the jump part of $Y$ is compound Poisson is depicted. When $Q_{t}>0$ and $X_{t}=0$, we see that $Q_{t}$ increases at rate $\left|d_{Y}\right|-1$, where $d_{Y}<-1$ is the drift of $Y$.

straightforward.) Apply (26) with $Y=\exp \left\{-\alpha t_{2}(0)+\beta t_{1}(0)\right\}$. Since $t_{1}(0)=\sup \{t \leq$ $\left.0: N_{1}(\{t\})=1\right\}, t_{2}(0)=\inf \left\{t>t_{1}(0): N_{2}(\{t\})=1\right\}$, and $\mathrm{P}_{N_{1}}\left(t_{1}(0)=0<t_{2}(0)\right)=1$, we have $t_{1}(0) \circ \theta_{t}=-t$ and $t_{2}(0) \circ \theta_{t}=t_{2}(0)-t, \mathrm{P}_{N_{1}}$-a.s. on $\left\{t_{1}(0)<t<t_{2}(0)\right\}$. Therefore, $Y \circ \theta_{t}=\exp \left\{-\alpha t_{2}(0)\right\} \mathrm{e}^{(\alpha-\beta) t}, \mathrm{P}_{N_{1}}$-a.s. on $\left\{t_{1}(0)<t<t_{2}(0)\right\}$, and so

$$
\lambda_{M} \mathrm{E}_{M}\left[\exp \left\{-\alpha t_{2}(0)+\beta t_{1}(0)\right\}\right]=\lambda \frac{\mathrm{E}_{N_{1}}\left[\exp \left\{-\beta t_{2}(0)\right\}\right]-\mathrm{E}_{N_{1}}\left[\exp \left\{-\alpha t_{2}(0)\right\}\right]}{\alpha-\beta} .
$$

Arguing in a similar manner, through the exchange formula between $M$ and $N_{2}$, we obtain

$$
\lambda_{M} \mathrm{E}_{M}\left[\exp \left\{-\beta t_{2}(0)+\alpha t_{1}(0)\right\}\right]=\lambda \frac{\mathrm{E}_{N_{2}}\left[\exp \left\{\alpha t_{1}(0)\right\}\right]-\mathrm{E}_{N_{2}}\left[\exp \left\{\beta t_{1}(0)\right\}\right]}{\beta-\alpha} .
$$


Setting $\beta=0$, and then $\alpha=0$, in (27) we obtain

$$
\begin{aligned}
& \lambda_{M} \mathrm{E}_{M}\left[\exp \left\{-\alpha t_{2}(0)\right\}\right]=\lambda \frac{1-\mathrm{E}_{N_{1}}\left[\exp \left\{-\alpha t_{2}(0)\right\}\right]}{\alpha}, \\
& \lambda_{M} \mathrm{E}_{M}\left[\exp \left\{\beta t_{1}(0)\right\}\right]=\lambda \frac{1-\mathrm{E}_{N_{1}}\left[\exp \left\{-\beta t_{2}(0)\right\}\right]}{\beta} .
\end{aligned}
$$

On the other hand, with $\alpha=0$ in (28), we have

$$
\lambda_{M} \mathrm{E}_{M}\left[\exp \left\{-\beta t_{2}(0)\right\}\right]=\lambda \frac{1-\mathrm{E}_{N_{2}}\left[\exp \left\{\beta t_{1}(0)\right\}\right]}{\beta} .
$$

The exchange formula between $N_{1}$ and $N_{2}$ shows that the right-hand sides of (30) and (31) are equal. Substituting these and (29) into (27) we obtain the result.

\subsection{Observed idle periods}

We are interested in the distribution of the idle period $(g(0), d(0))$, given that $Q_{0}=0$. The rationale used for this computation is as follows. We can always assume that $X_{0}>0$, since this is an event with probability 1 . If $Q_{0}=0$ then $Q_{t}$ will remain 0 at least until $X$ hits 0 , since $Q$ cannot increase unless there is an accumulation of local time $L$, and this can happen only when $X$ is 0 . Recall that the first hitting time of 0 by $X$ is denoted by $D=\inf \left\{t>0: X_{t}=0\right\}$. If $Q_{0}=0$, the first time that $Q$ becomes positive has been denoted by $d(0)$. Our claim is as follows.

Lemma 3. Given that $Q_{0}=0$, the ending time of the idle period is a.s. equal to D, i.e.

$$
\mathrm{P}\left(d(0)=D \mid Q_{0}=0\right)=1 .
$$

Proof. From the argument above we have $D \leq d(0)$ a.s. on $\left\{Q_{0}=0\right\}$. Suppose that there is $\Omega_{0} \subset \Omega$ with $\mathrm{P}\left(\Omega_{0}\right)>0$ such that $Q_{0}=0$ and $D<d(0)$ a.s. on $\Omega_{0}$. If $Q_{0}=0$ and $D<d(0)$ then

$$
Q_{t}=\sup _{D \leq u \leq t}\{L(u, t]-(t-u)\} \equiv 0 \quad \text { for all } t \in(D, d(0)) .
$$

This implies that

$$
L(u, t] \leq t-u \text { for all } D<u<t<d(0),
$$

which means that, for $\omega \in \Omega_{0}, L(\omega, \cdot)$ is absolutely continuous on some right neighbourhood of $D$. If $Y$ is spectrally negative or if $Y$ is spectrally positive but not of bounded variation, then $L$ is a.s. singular on any right neighbourhood of $D$, and we obtain a contradiction. If $Y$ is spectrally positive with bounded variation paths then $L$ is absolutely continuous and is given by (15). In this case, $Q$ increases at rate $\left|d_{Y}\right|-1>0$ whenever it is positive, and this immediately shows that here, too, $D=d(0)$ a.s. on $\left\{Q_{0}=0\right\}$.

Remark 1. The result in Lemma 3 can alternatively be expressed by saying that the process $\left(L_{t}-t: t \geq 0\right)$ is under $\mathrm{P}_{0}$ initially increasing. In [20, Proposition 6.3] this was proved for the case in which $X$ is a reflecting Brownian motion, and the proof therein could have been modified to cover the present case. However, we found that it motivated us to give the above proof which highlights other aspects not given in [20]. 
Using Lemma 2, we shall reduce the problem to that of finding the distribution of $D=$ $\inf \left\{t>0: X_{t}=0\right\}$ given that $Q_{0}=0$. Let $N_{1}$ and $N_{2}$ be the point processes with points $\{g(n)\}$ and $\{d(n)\}$, respectively. Then $M(\mathrm{~d} t) / \mathrm{d} t=\mathbf{1}\left(Q_{t}=0\right)$, and so

$$
\mathrm{P}_{M}=\mathrm{P}\left(\cdot \mid Q_{0}=0\right) \text {. }
$$

Equation (25), together with Lemma 3, then gives

$$
\mathrm{E}\left[\mathrm{e}^{-\alpha d(0)+\beta g(0)} \mid Q_{0}=0\right]=\frac{\alpha \mathrm{E}\left[\mathrm{e}^{-\alpha D} \mid Q_{0}=0\right]-\beta \mathrm{E}\left[\mathrm{e}^{-\beta D} \mid Q_{0}=0\right]}{\alpha-\beta} .
$$

To compute the distribution of $D$ given $Q_{0}=0$, we need the following two lemmas.

Lemma 4. Let

$$
G:=\sup \left\{t<0: X_{t}=0\right\}
$$

Then it holds that

$$
\left\{Q_{0}=0\right\}=\left\{Q_{G}+G \leq 0\right\} .
$$

Proof. Since $X_{t}>0$ for all $t \in(G, D)$, we have

$$
L(s, t]=0, \quad G \leq s \leq t \leq D .
$$

Recall that

$$
Q_{t}=\mathcal{R}_{s, t} \hat{L}\left(Q_{s}\right)=\sup _{s \leq u \leq t} \hat{L}(u, t] \vee\left(Q_{s}+\hat{L}(s, t]\right) .
$$

So, if $G \leq s \leq t \leq D$, we have $\hat{L}(s, t]:=L(s, t]-(t-s)=-(t-s)$, i.e.

$$
Q_{t}=\left(Q_{s}-(t-s)\right)^{+}, \quad G \leq s \leq t \leq D .
$$

If we assume that $Q_{0}=0$, we have $G \leq g(0)$ and so

$$
0=Q_{g(0)}=\left(Q_{G}-(g(0)-G)\right)^{+},
$$

which implies that $Q_{G}+G=g(0) \leq 0$.

Lemma 5. (i) Conditional on $X_{0}$, the random variables $Q_{G}, G$, and $D$ are independent (under $\mathrm{P}$ ).

(ii) For all $x \geq 0$ and $t \geq 0$,

$$
\mathrm{P}\left(Q_{G}>t\right)=\mathrm{P}_{x}\left(Q_{G}>t\right)=\mathrm{P}_{0}\left(Q_{0}>t\right)=\mathrm{e}^{-\theta^{*} t},
$$

where $\theta^{*}=\psi_{Y}(1)$ if $Y$ is spectrally negative or is equal to the unique positive solution of $\theta^{*}=\psi_{Y}\left(\theta^{*}\right)$ if $Y$ is spectrally positive.

(iii) $Q_{G}$ is independent of $(G, D)$ (under $\left.\mathrm{P}\right)$.

Proof. (i) The independence follows from the strong Markov property at $G$ (at which time $X_{G}=0$ ) and the Markov property at 0 . Indeed, first observe that $G$ is a stopping time with respect to the filtration $\left\{\widetilde{F}_{t}:=\sigma\left(X_{-s}, 0 \leq s \leq t\right), t \geq 0\right\}$. Second, $Q_{G}=\widetilde{\mathcal{R}}_{G} \hat{L}=$ $\sup _{s \leq G} \hat{L}(s, G]=\sup _{s \leq G}(L(s, G]-(G-s))$ and so $Q_{G} \mathbf{1}(G<t)$ is measurable with respect to $\mathcal{F}_{t}^{\prime}:=\sigma\left(X_{-s}, s>t\right)$ for all $t$. This proves independence between $Q_{G}$ and $G$. Third, $D$ is measurable with respect to $\mathcal{F}_{0}^{\prime \prime}=\sigma\left(X_{s}, s \geq 0\right)$. So, conditionally on $X_{0}$, the random variable 
$D$ is independent of the pair $\left(Q_{G}, G\right)$. (ii) The distribution statement about $Q_{G}$ follows from the strong Markov property at $G$. Let, as usual, $\mathcal{F}_{-G}=\left\{A \in \sigma\left(X_{-s}, s \geq 0\right): A \cap\{-G \leq\right.$ $\left.t\} \in \mathcal{F}_{-t}\right\}$. Since $Q_{G}=Q_{0} \circ \theta_{G}$,

$$
\begin{aligned}
\mathrm{P}\left(Q_{G}>t\right) & =\mathrm{P}\left(Q_{0} \circ \theta_{G}>t\right) \\
& =\mathrm{E}\left[\mathrm{P}\left(Q_{0} \circ \theta_{G}>t \mid \mathcal{F}_{-G}\right)\right] \\
& =\mathrm{E}\left[\mathrm{P}_{X_{G}}\left(Q_{0}>t\right)\right] \\
& =\mathrm{P}_{0}\left(Q_{0}>t\right) \\
& =\mathrm{e}^{-\theta^{*} t},
\end{aligned}
$$

where the latter follows from Theorem 3. (iii) This is immediate from (i) and (ii).

Proposition 5. (Distribution of the observed idle period.) Fix $\alpha, \beta \geq 0, \alpha \neq \beta$.

(i) When $Y$ is spectrally negative, we have

$$
\begin{aligned}
& \mathrm{E}\left[\mathrm{e}^{-\alpha d(0)+\beta g(0)} \mid Q_{0}=0\right] \\
& \quad=\frac{\Phi_{Y}(0)}{1-\Phi_{Y}(0)} \frac{\psi_{Y}(1)}{\alpha-\beta}\left(\frac{\alpha}{\alpha-\psi_{Y}(1)} \frac{\Phi_{Y}(\alpha)-1}{\Phi_{Y}(\alpha)}-\frac{\beta}{\beta-\psi_{Y}(1)} \frac{\Phi_{Y}(\beta)-1}{\Phi_{Y}(\beta)}\right) .
\end{aligned}
$$

(ii) When $Y$ is spectrally positive, we have

$$
\mathrm{E}\left[\mathrm{e}^{-\alpha d(0)+\beta g(0)} \mid Q_{0}=0\right]=\frac{\psi_{Y}^{\prime}(0+)}{1-\psi_{Y}^{\prime}(0+)} \frac{\theta^{*}}{\alpha-\beta}\left(\frac{\alpha-\Phi_{Y}(\alpha)}{\alpha-\theta^{*}}-\frac{\beta-\Phi_{Y}(\beta)}{\beta-\theta^{*}}\right),
$$

where $\theta^{*}>0$ is defined by $\psi_{Y}\left(\theta^{*}\right)=\theta^{*}$.

Proof. From Lemma 5, $D$ and $Q_{G}$ are conditionally independent given $X_{0}$ and $G$. Hence,

$$
\begin{aligned}
\mathrm{E}\left[\mathrm{e}^{-\theta D} \mathbf{1}\left(Q_{G}+G \leq 0\right) \mid X_{0}, G\right] & =\mathrm{E}\left[\mathrm{e}^{-\theta D} \mid X_{0}, G\right] \mathrm{P}\left(Q_{G} \leq-G \mid X_{0}, G\right) . \\
& =\mathrm{E}\left[\mathrm{e}^{-\theta D} \mid X_{0}, G\right]\left(1-\mathrm{e}^{\theta^{*} G}\right) \\
& =\mathrm{E}\left[\mathrm{e}^{-\theta D}-\mathrm{e}^{-\theta D+\theta^{*} G} \mid X_{0}, G\right],
\end{aligned}
$$

where the second line was obtained from the facts (all consequences of Lemma 5) that (i) $D$ and $G$ are conditionally independent given $X_{0}$, (ii) $Q_{G}$ and $G$ are also conditionally independent given $X_{0}$, and (iii) $Q_{G}$ is independent of $X_{0}$ and exponentially distributed with parameter

$$
\theta^{*}= \begin{cases}\psi_{Y}(1) & \text { if } Y \text { is spectrally negative } \\ \psi_{Y}\left(\theta^{*}\right) & \text { if } Y \text { is spectrally positive }\end{cases}
$$

Taking expectations we obtain

$$
\mathrm{E}\left[\mathrm{e}^{-\theta D} \mathbf{1}\left(Q_{G}+G \leq 0\right)\right]=\mathrm{E}\left[\mathrm{e}^{-\theta D}-\mathrm{e}^{-\theta D+\theta^{*} G}\right],
$$

and, using Lemma 4,

$$
\mathrm{E}\left[\mathrm{e}^{-\theta D} \mid Q_{0}=0\right]=\frac{\mathrm{E}\left[\mathrm{e}^{-\theta D}\right]-\mathrm{E}\left[\mathrm{e}^{-\theta D+\theta^{*} G}\right]}{\mathrm{P}\left(Q_{0}=0\right)} .
$$


We now use a version of Lemma 2, formulated for excursions of general stationary processes; see Pitman [21, p. 298] and the references therein. The random measures $N_{1}$ and $N_{2}$ correspond to the beginnings and ends of excursions of the stationary process $\left(X_{t}, t \in \mathbb{R}\right)$, and, since there is never an interval of time over which $X$ is 0 , the random measure $M$ coincides with the Lebesgue measure, while $\mathrm{P}_{M}=\mathrm{P}$. Applying Pitman's result (an analogue of (25)) gives

$$
\mathrm{E}\left[\mathrm{e}^{-\alpha D+\beta G}\right]=\frac{\alpha \mathrm{E}\left[\mathrm{e}^{-\alpha D}\right]-\beta \mathrm{E}\left[\mathrm{e}^{-\beta D}\right]}{\alpha-\beta} .
$$

The joint Laplace transform of $D$ and $G$ is thus expressible in terms of the Laplace transform of $D$. Combining the last two displays we obtain

$$
\mathrm{E}\left[\mathrm{e}^{-\theta D} \mid Q_{0}=0\right]=\frac{1}{\mathrm{P}\left(Q_{0}=0\right)} \frac{\theta^{*}}{\theta-\theta^{*}}\left(\mathrm{E}\left[\mathrm{e}^{-\theta^{*} D}\right]-\mathrm{E}\left[\mathrm{e}^{-\theta D}\right]\right) .
$$

Using this in (32) results in

$$
\begin{aligned}
\mathrm{E}\left[\mathrm{e}^{-\alpha d(0)+\beta g(0)} \mid Q_{0}=0\right] \\
=\frac{1}{\mathrm{P}\left(Q_{0}=0\right)} \frac{\theta^{*}}{\alpha-\beta} \\
\quad \times\left(\frac{\alpha}{\alpha-\theta^{*}}\left(\mathrm{E}\left[\mathrm{e}^{-\theta^{*} D}\right]-\mathrm{E}\left[\mathrm{e}^{-\alpha D}\right]\right)-\frac{\beta}{\beta-\theta^{*}}\left(\mathrm{E}\left[\mathrm{e}^{-\theta^{*} D}\right]-\mathrm{E}\left[\mathrm{e}^{-\beta D}\right]\right)\right) .
\end{aligned}
$$

So far, the arguments are general and hold for both spectrally negative and positive Lévy processes $Y$, as long as $\theta^{*}$ is taken as in (33). Next, substituting the expression for the Laplace transform of $D$ from (10) and (18) for the spectrally negative, respectively, positive cases, we obtain the result.

\subsection{Observed busy periods}

In this section we follow the ideas of [23]. We are interested in the distribution of the observed busy period, as defined in (24). On the conditioning event $\left\{Q_{0}>0\right\}$, we have, by our enumeration convention,

$$
g(0)<d(0)<0<g(1), \quad \text { P-a.s. }
$$

Using Lemma 2 with $N_{1}$ or $N_{2}$, the point process with points $\{d(n)\}$ or, respectively, $\{g(n\})$, we have

$$
\begin{aligned}
& \mathrm{E}\left[\mathrm{e}^{-\alpha g(1)+\beta d(0)} \mid Q_{0}>0\right] \\
& \quad=\frac{\alpha \mathrm{E}\left[\mathrm{e}^{-\alpha g(1)} \mid Q_{0}>0\right]-\beta \mathrm{E}\left[\mathrm{e}^{-\beta g(1)} \mid Q_{0}>0\right]}{\alpha-\beta}, \quad \alpha, \beta>0 .
\end{aligned}
$$

Recall the evolution equation for $Q$ :

$$
Q_{t}=Q_{s}+L(s, t]-(t-s)-\inf _{s \leq u \leq t}\left\{Q_{s}+L(s, u]-(u-s)\right\} .
$$

Let $s=0$, and assume that $Q_{0}>0$. Since $X_{0}>0$, P-a.s., we have $L(0, t]=0$ for all $0<t<D=\inf \left\{r>0: X_{r}=0\right\}$, and so,

$$
Q_{t}=Q_{0}-t-\inf _{0 \leq u \leq t}\left\{Q_{0}-u\right\}=Q_{0}-t \quad \text { a.s. on }\left\{Q_{0}>0, t<D\right\},
$$


which implies that

$$
g(1)= \begin{cases}Q_{0} & \text { a.s. on }\left\{0<Q_{0}<D\right\} \\ g(1) \circ \theta_{D} & \text { a.s. on }\left\{Q_{0}>D\right\}\end{cases}
$$

Now, if $Q_{0}>D$, we have $Q_{D-}=Q_{0}-D$, so from (36), $Q$ evolves as

$$
Q_{D+t}=Q_{0}-D+L[D, D+t]-t, \quad t \geq 0,
$$

as long as $Q_{D+t}>0$. This implies that, a.s. on $\left\{Q_{0}>D\right\}$,

$$
g(1) \circ \theta_{D}-D=\inf \left\{t>0: Q_{0}-D+L[D, D+t]-t=0\right\} .
$$

Therefore, (37) becomes

$$
g(1)= \begin{cases}Q_{0} & \text { a.s. on }\left\{0<Q_{0}<D\right\} \\ D+\inf \left\{t>0: Q_{0}-D+L[D, D+t]-t=0\right\} & \text { a.s. on }\left\{Q_{0}>D\right\}\end{cases}
$$

Now consider the inverse local-time process, with the origin of time placed at $D$, i.e.

$$
L_{D ; x}^{-1}:=\inf \{t>0: L[D, D+t]>x\}, \quad x \geq 0 .
$$

By the strong Markov property for $X$ at the stopping time $D$ we find that the P-distribution of $\left(L_{D ; x}^{-1}, x \geq 0\right)$ is the same as the $\mathrm{P}_{0}$-distribution of $\left(L_{x}^{-1}, x \geq 0\right)$, which has been identified in Propositions 2 and 4. Thus, $\left(L_{D ; x}^{-1}, x \geq 0\right)$ is a (proper) subordinator. Next consider the spectrally negative Lévy process

$$
\tilde{\Lambda}_{x}:=x-L_{D ; x}^{-1}, \quad x \geq 0 .
$$

Note that $\mathrm{P}\left(\tilde{\Lambda}_{0}=0\right)$. The Laplace exponent of $\tilde{\Lambda}$ is the function $\psi_{\Lambda}$ of $(21)$. Define the hitting time of level $-a$ by $\tilde{\Lambda}$ :

$$
\sigma(\tilde{\Lambda} ; a):=\inf \left\{x>0: \tilde{\Lambda}_{x}<-a\right\}, \quad a>0,
$$

Equation (55), below, gives us the Laplace transform of $\sigma(\tilde{\Lambda} ; a)$ in terms of the scale functions of $\tilde{\Lambda}$, defined in (52) and (53), below. Combining them we obtain

$$
\int_{0}^{\infty} \mathrm{e}^{-\theta a} \mathrm{E}\left[\mathrm{e}^{-q \sigma(\tilde{\Lambda} ; a)}\right] \mathrm{d} a=\frac{1}{\psi_{\Lambda}(\theta)-q}\left(\frac{\psi_{\Lambda}(\theta)}{\theta}-\frac{q}{\Phi_{\Lambda}(\theta)}\right)=: H^{(q)}(\theta) .
$$

As can be easily seen from Lemma 1 , for any $a>0$,

$$
\inf \{t>0: t-L[D, D+t] \geq a\}=\inf \left\{x>0: \tilde{\Lambda}_{x}<-a\right\}+a=\sigma(\tilde{\Lambda} ; a)+a .
$$

Using this in (38), we obtain

$$
g(1)= \begin{cases}Q_{0} & \text { a.s. on }\left\{0<Q_{0}<D\right\} \\ Q_{0}+\sigma\left(\tilde{\Lambda} ; Q_{0}-D\right) & \text { a.s. on }\left\{Q_{0}>D\right\}\end{cases}
$$

It is useful to keep in mind that $\tilde{\Lambda}$ is independent of $Q_{0}-D$, by the strong Markov property of $X$ at $D$. We are now ready to compute the Laplace transform appearing on the right-hand side 
of (35):

$$
\begin{aligned}
\mathrm{E}\left[\mathrm{e}^{-\alpha g(1)} ; Q_{0}>0\right]= & \mathrm{E}\left[\mathrm{e}^{-\alpha g(1)} ; 0<Q_{0}<D\right]+\mathrm{E}\left[\mathrm{e}^{-\alpha g(1)} ; Q_{0}>D\right] \\
= & \mathrm{E}\left[\exp \left\{-\alpha Q_{0}\right\} ; 0<Q_{0}<D\right] \\
& +\mathrm{E}\left[\exp \left\{-\alpha\left(Q_{0}+\sigma\left(\tilde{\Lambda} ; Q_{0}-D\right)\right)\right\} ; Q_{0}>D\right] \\
= & \mathrm{E}\left[\exp \left\{-\alpha Q_{0}\right\} ; Q_{0}>0\right] \\
& -\mathrm{E}\left[\exp \left\{-\alpha Q_{0}\right\}\left(1-\exp \left\{-\alpha \sigma\left(\tilde{\Lambda} ; Q_{0}-D\right)\right\}\right) ; Q_{0}>D\right] .
\end{aligned}
$$

Recall that $\mathrm{P}\left(Q_{0}>x\right)=\mu \mathrm{e}^{-\theta^{*} x}$, and so

$$
\mathrm{E}\left[\exp \left\{-\alpha Q_{0}\right\} ; Q_{0}>0\right]=\mu \frac{\theta^{*}}{\alpha+\theta^{*}} .
$$

To compute the second and third terms, we need some elementary properties of exponentially distributed random variables, which we state without proof.

Lemma 6. Let $T$ be an exponentially distributed random variable with parameter $\lambda$, and let $(X, Y), X \geq 0, Y \geq 0$, be a two-dimensional random variable independent of $T$. Then $X$ and $T-X-Y$ are independent given $T>X+Y$. Moreover,

$$
\begin{gathered}
\mathrm{E}\left[\mathrm{e}^{-\alpha(T-X-Y)} \mid T>X+Y\right]=\frac{\lambda}{\alpha+\lambda}, \\
\mathrm{E}\left[\mathrm{e}^{-\alpha X} ; T>X+Y\right]=\mathrm{E}\left[\mathrm{e}^{-(\alpha+\lambda) X-\lambda Y}\right] .
\end{gathered}
$$

Use (36) once more with $s=G=\sup \left\{t<0: X_{t}=0\right\}$, and $t=0$, taking into account the fact that $L$ is not supported on $(G, 0)$, to obtain $Q_{0}=Q_{G}+G$ a.s. on $\left\{Q_{0}>0\right\}$. Since $Q_{G}$ is exponentially distributed with parameter $\theta^{*}$ and independent of $(G, D)$ (from Lemma 5), we have, applying Lemma 6, the following result.

Lemma 7. Given $Q_{0}>D$, the random variables $Q_{0}-D$ and $D$ are independent. Moreover,

$$
\begin{gathered}
\mathrm{E}\left[\exp \left\{-\alpha\left(Q_{0}-D\right)\right\} \mid Q_{0}>D\right]=\frac{\theta^{*}}{\alpha+\theta^{*}}, \\
\mathrm{E}\left[\mathrm{e}^{-\alpha D} ; Q_{0}>D\right]=\mathrm{E}\left[\mathrm{e}^{-\left(\alpha+\theta^{*}\right) D+\theta^{*} G}\right] .
\end{gathered}
$$

Using Lemma 7, we write the last term of (40) as follows:

$$
\begin{aligned}
\mathrm{E}[\exp \{ & \left.\left.-\alpha Q_{0}\right\}\left(1-\exp \left\{-\alpha \sigma\left(\tilde{\Lambda} ; Q_{0}-D\right)\right\}\right) ; Q_{0}>D\right] \\
= & \mathrm{P}\left(Q_{0}>D\right) \mathrm{E}\left[\mathrm{e}^{-\alpha D} \exp \left\{-\alpha\left(Q_{0}-D\right)\right\}\left(1-\exp \left\{-\alpha \sigma\left(\tilde{\Lambda} ; Q_{0}-D\right)\right\}\right) \mid Q_{0}>D\right] \\
= & \mathrm{P}\left(Q_{0}>D\right) \mathrm{E}\left[\mathrm{e}^{-\alpha D} \mid Q_{0}>D\right] \\
& \times \mathrm{E}\left[\exp \left\{-\alpha\left(Q_{0}-D\right)\right\}\left(1-\exp \left\{-\alpha \sigma\left(\tilde{\Lambda} ; Q_{0}-D\right)\right\}\right) \mid Q_{0}>D\right] \\
= & \mathrm{E}\left[\mathrm{e}^{-\left(\alpha+\theta^{*}\right) D+\theta^{*} G}\right] \mathrm{E}\left[\mathrm{e}^{-\alpha V}\left(1-\mathrm{e}^{-\alpha \sigma(\tilde{\Lambda} ; V)}\right)\right],
\end{aligned}
$$

where, in the last term, we introduced a random variable $V$, exponentially distributed with parameter $\theta^{*}$, independent of everything else (due to the fact that $Q_{0}-D$, conditionally on being positive, is exponential with parameter $\theta^{*}$, independent of $\tilde{\Lambda}$ ). The first term of (42) can be computed as in (34). We have, for all $\alpha, \beta \geq 0, \alpha \neq \beta$,

$$
\mathrm{E}\left[\mathrm{e}^{-\alpha D+\beta G}\right]=\frac{\Phi_{Y}(0)}{\alpha-\beta}\left(\frac{\alpha}{\Phi_{Y}(\alpha)}-\frac{\beta}{\Phi_{Y}(\beta)}\right),
$$


and, for the spectrally positive case, for all $\alpha, \beta \geq 0, \alpha \neq \beta$,

$$
\mathrm{E}\left[\mathrm{e}^{-\alpha D+\beta G}\right]=\psi_{Y}^{\prime}(0+) \frac{\Phi_{Y}(\alpha)-\Phi_{Y}(\beta)}{\alpha-\beta} .
$$

Note that taking account of the definition of $\psi_{\Lambda}$, (21), for both the spectrally negative and positive cases, and the fact that $\psi_{\Lambda}\left(\theta^{*}\right)=0$, we see that generically for both cases, for all $\alpha \geq 0$

$$
\mathrm{E}\left[\mathrm{e}^{-\left(\alpha+\theta^{*}\right) D+\theta^{*} G}\right]=\frac{\mu}{\alpha}\left(\alpha-\psi_{\Lambda}\left(\alpha+\theta^{*}\right)\right) .
$$

For the second term of (42), we have, using (39),

$$
\begin{aligned}
\mathrm{E}\left[\mathrm{e}^{-\alpha V}\left(1-\mathrm{e}^{-\alpha \sigma(\tilde{\Lambda} ; V)}\right)\right] & =\frac{\theta^{*}}{\alpha+\theta^{*}}-\theta^{*} \int_{0}^{\infty} \mathrm{e}^{-\theta^{*} v} \mathrm{e}^{-\alpha v} \mathrm{E}\left[\mathrm{e}^{-\alpha \sigma(\tilde{\Lambda} ; v)}\right] \mathrm{d} v \\
& =\frac{\theta^{*}}{\alpha+\theta^{*}}-\theta^{*} H^{(\alpha)}\left(\alpha+\theta^{*}\right) \\
& =\frac{\theta^{*}}{\alpha+\theta^{*}}-\frac{\theta^{*}}{\psi_{\Lambda}\left(\alpha+\theta^{*}\right)-\alpha}\left(\frac{\psi_{\Lambda}\left(\alpha+\theta^{*}\right)}{\alpha+\theta^{*}}-\frac{\alpha}{\Phi_{\Lambda}(\alpha)}\right) \\
& =\frac{\theta^{*}}{\alpha+\theta^{*}} \frac{\alpha+\theta^{*}-\Phi_{\Lambda}(\alpha)}{\Phi_{\Lambda}(\alpha)} \frac{\alpha}{\psi_{\Lambda}\left(\alpha+\theta^{*}\right)-\alpha} .
\end{aligned}
$$

Multiplying (43) and (44) we obtain the following expression for (42):

$$
\mathrm{E}\left[\exp \left\{-\alpha Q_{0}\right\}\left(1-\exp \left\{-\alpha \sigma\left(\tilde{\Lambda} ; Q_{0}-D\right)\right\}\right) ; Q_{0}>D\right]=\frac{\mu \theta^{*}}{\alpha+\theta^{*}}-\frac{\mu \theta^{*}}{\Phi_{\Lambda}(\alpha)} .
$$

This, together with (40) and (41), yields

$$
\mathrm{E}\left[\mathrm{e}^{-\alpha g(1)} \mid Q_{0}>0\right]=\frac{\theta^{*}}{\Phi_{\Lambda}(\alpha)} .
$$

Using (35), we finally come to rest at the following main result.

Proposition 6. (Distribution of the observed busy period.) For $\alpha, \beta>0, \alpha \neq \beta$,

$$
\mathrm{E}\left[\mathrm{e}^{-\alpha g(1)+\beta d(0)} \mid Q_{0}>0\right]=\frac{\theta^{*}}{\alpha-\beta}\left(\frac{\alpha}{\Phi_{\Lambda}(\alpha)}-\frac{\beta}{\Phi_{\Lambda}(\beta)}\right),
$$

where $\Phi_{\Lambda}$ is the right inverse of $\psi_{\Lambda}$, which is given in (21), and (cf. Theorem 3)

(i) $\theta^{*}>0$ is defined by $\psi_{Y}(1)$ in the case that $Y$ is spectrally negative,

(ii) $\theta^{*}>0$ is defined by $\psi_{Y}\left(\theta^{*}\right)=\theta^{*}$ in the case that $Y$ is spectrally positive.

\subsection{Typical idle and busy periods}

We now consider the problem of identifying the distribution of a typical idle and a typical busy period of $Q$. We place the origin of time at the beginning of such a period, by considering the appropriate Palm probability. Let $N_{g}$ and $N_{d}$ be the point processes with points $\{g(n)\}$, the beginnings of idle periods, and $\{d(n)\}$, the beginnings of busy periods, respectively. Let $\mathrm{P}_{g}$ and $\mathrm{P}_{d}$ be the Palm probability with respect to $N_{g}$ and, respectively, $N_{d}$. See Figure 4. 


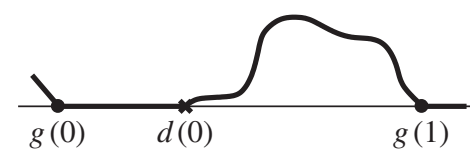

FigURE 4.

Using (29), we have

$$
\frac{1-\mu}{\lambda} \alpha \mathrm{E}\left[\mathrm{e}^{-\alpha d(0)} \mid Q_{0}=0\right]=1-\mathrm{E}_{g}\left[\mathrm{e}^{-\alpha d(0)}\right]
$$

where $\mu=\mathrm{P}\left(Q_{0}>0\right)$ and $\lambda$ is the common rate of $N_{g}$ and $N_{d}$. The right-hand side is precisely what we need. Everything on the left-hand side is known (see Proposition 5) except the rate $\lambda$. First consider the case when $Y$ is spectrally negative. Using Proposition 5(i) with $\beta=0$, we obtain

$$
\frac{1-\mu}{\lambda} \frac{\Phi_{Y}(0)}{1-\Phi_{Y}(0)} \psi_{Y}(1) \frac{\alpha}{\alpha-\psi_{Y}(1)} \frac{\Phi_{Y}(\alpha)-1}{\Phi_{Y}(\alpha)}=1-\mathrm{E}_{g}\left[\mathrm{e}^{-\alpha d(0)}\right] .
$$

Taking limits as $\alpha \rightarrow \infty$ (and since $\Phi_{Y}(\alpha) \rightarrow \infty$ ) we find the value of $\lambda$ and, so, the Laplace transform $\mathrm{E}_{g}\left[\mathrm{e}^{-\alpha d(0)}\right]$ of the typical idle period. The result is given in Proposition 7(i), below.

We repeat the procedure for the spectrally positive case and, using Proposition 5(ii), we obtain

$$
\frac{1-\mu}{\lambda} \frac{\psi_{Y}^{\prime}(0)}{1-\psi_{Y}^{\prime}(0)} \theta^{*} \frac{\alpha-\Phi_{Y}(\alpha)}{\alpha-\theta^{*}}=1-\mathrm{E}_{g}\left[\mathrm{e}^{-\alpha d(0)}\right] .
$$

Note that $\lim _{\theta \rightarrow \infty} \psi_{Y}(\theta) / \theta=\infty$ if $Y$ is of unbounded variation, and so $\lim _{\alpha \rightarrow \infty} \Phi_{Y}(\alpha) / \alpha=0$. Thus, we can find $\lambda$ and $\mathrm{E}_{g}\left[\mathrm{e}^{-\alpha d(0)}\right]$; see Proposition (7)(ii), below.

But if $Y$ is spectrally positive (with nonmonotone paths) and of bounded variation then $L$ is absolutely continuous and has a drift $d_{Y}$; see (15). We can easily see, e.g. from (14), that

$$
\psi_{Y}(\theta)=\log \mathrm{E}\left[\exp \left\{-\theta\left(Y_{1}-Y_{0}\right)\right\}\right]=\left|d_{Y}\right| \theta-\int_{0}^{\infty}\left(1-\mathrm{e}^{-\theta y}\right) \Pi(\mathrm{d} y),
$$

and, since $\int_{0}^{\infty}(y \wedge 1) \Pi(\mathrm{d} y)<\infty$, we obtain $\lim _{\theta \rightarrow \infty} \psi_{Y}(\theta) / \theta=\left|d_{Y}\right|$. So $\lim _{\alpha \rightarrow \infty} \Phi_{Y}(\alpha) / \alpha$ $=1 /\left|d_{Y}\right|$. Again, we can find $\lambda$ and $\mathrm{E}_{g}\left[\mathrm{e}^{-\alpha d(0)}\right]$; see Proposition (7)(iii), below.

Proposition 7. (Distribution of the typical idle period.) Fix $\alpha>0$. Let $\mathrm{P}_{g}$ be the Palm probability with respect to the beginnings of the idle periods of $Q$.

(i) When $Y$ is spectrally negative, we have

$$
\lambda=\Phi_{Y}(0) \psi_{Y}(1), \quad \mathrm{E}_{g}\left[\mathrm{e}^{-\alpha d(0)}\right]=1-\frac{\alpha}{\Phi_{Y}(\alpha)} \frac{\Phi_{Y}(\alpha)-1}{\alpha-\psi_{Y}(1)} .
$$

(ii) When $Y$ is spectrally positive and $\int_{0}^{1} y \Pi(\mathrm{d} y)=\infty$, we have

$$
\lambda=\psi_{Y}^{\prime}(0) \theta^{*}, \quad \mathrm{E}_{g}\left[\mathrm{e}^{-\alpha d(0)}\right]=1-\frac{\alpha-\Phi_{Y}(\alpha)}{\alpha-\theta^{*}},
$$

where $\theta^{*}>0$ satisfies $\theta^{*}=\psi_{Y}\left(\theta^{*}\right)$.

(iii) When $Y$ is spectrally positive and $\int_{0}^{1} y \Pi(\mathrm{d} y)<\infty$, we have

$$
\lambda=\psi_{Y}^{\prime}(0) \theta^{*}\left(1-\frac{1}{\left|d_{Y}\right|}\right), \quad \mathrm{E}_{g}\left[\mathrm{e}^{-\alpha d(0)}\right]=1-\frac{\left|d_{Y}\right|}{\left|d_{Y}\right|-1} \frac{\alpha-\Phi_{Y}(\alpha)}{\alpha-\theta^{*}},
$$

where $\theta^{*}>0$ satisfies $\theta^{*}=\psi_{Y}\left(\theta^{*}\right)$ and $d_{Y}$ is the drift defined in (14)-(15). 
Remark 2. By Assumption (A2) (see Section 3) we have $d_{Y}<-1$ and so the constant above is positive.

In the same vein, we obtain the Laplace transform $\mathrm{E}_{d}\left[\mathrm{e}^{-\alpha g(1)}\right]$ of a typical busy period. Note that, under $\mathrm{P}_{d}$, we have $d(0)=0$, and so the first busy period to the right of the origin of time is the interval $(d(0), g(1))$. We have

$$
\frac{\mu}{\lambda} \alpha \mathrm{E}\left[\mathrm{e}^{-\alpha g(1)} \mid Q_{0}>0\right]=1-\mathrm{E}_{d}\left[\mathrm{e}^{-\alpha g(1)}\right] .
$$

Using Proposition 6 with $\beta=0$, we have

$$
\frac{\mu \theta^{*}}{\lambda} \frac{\alpha}{\Phi_{\Lambda}(\alpha)}=1-\mathrm{E}_{d}\left[\mathrm{e}^{-\alpha g(1)}\right] .
$$

From the expression for $\psi_{\Lambda}(\theta)$, (21), we find that $\lim _{\theta \rightarrow \infty} \psi_{\Lambda}(\theta) / \theta=1$. So,

$$
\lim _{\alpha \rightarrow \infty} \frac{\alpha}{\Phi_{\Lambda}(\alpha)}=1
$$

Therefore, we have the following proposition.

Proposition 8. (Distribution of the typical busy period.) Fix $\alpha>0$. Let $\mathrm{P}_{d}$ be the Palm probability with respect to the beginnings of the busy periods of $Q$. Let $\psi_{\Lambda}$ be defined as in (21), and let $\Phi_{\Lambda}$ be its right inverse function. Then

$$
\lambda=\mu \theta^{*}, \quad \mathrm{E}_{d}\left[\mathrm{e}^{-\alpha g(1)}\right]=1-\frac{\alpha}{\Phi_{\Lambda}(\alpha)},
$$

where $\mu=\Phi_{Y}(0)$ and $\theta^{*}=\psi_{Y}(1)$ if $Y$ is spectrally negative, and $\mu=\psi_{Y}^{\prime}(0)$ and $\theta^{*}>0$ is defined through $\theta^{*}=\psi_{Y}\left(\theta^{*}\right)$ if $Y$ is spectrally positive.

Corollary 1. The mean duration of a typical idle period is $(1-\mu) / \lambda$, while the mean duration of a typical busy period is $\mu / \lambda$, where $\mu$ is given by (19) and $\lambda$ is given in Propositions 7 and 8 .

Remark 3. By the relation between $\mathrm{P}$ and the Palm probability $\mathrm{P}_{d}$, it follows that the typical idle and busy periods are stochastically smaller than the observed idle and busy periods, respectively. This can be seen from Equation (1.4.2) of [1, p. 33]. In particular, the means of the former are shorter than the means of the latter. (This is usually referred to as the 'inspection paradox'.) This gives us several inequalities between different quantities associated with the Lévy process $Y$. To give an example, we compare the mean durations of idle periods in the spectrally negative case. From Proposition 5 we have

$$
\begin{aligned}
\mathrm{E}\left[\mathrm{e}^{-\alpha d(0)} \mid Q_{0}=0\right] & =\frac{\Phi_{Y}(0) \psi_{Y}(1)}{1-\Phi_{Y}(0)}\left(\frac{\Phi_{Y}(\alpha)-1}{\Phi_{Y}(\alpha)\left(\alpha-\psi_{Y}(1)\right)}\right) \\
& =: \frac{\Phi_{Y}(0) \psi_{Y}(1)}{1-\Phi_{Y}(0)} F(\alpha) .
\end{aligned}
$$

It follows that

$$
\mathrm{E}\left[d(0) \mid Q_{0}=0\right]=-\frac{\Phi_{Y}(0) \psi_{Y}(1)}{1-\Phi_{Y}(0)} F^{\prime}(0) .
$$

Since $d(0)$ and $-g(0)$ are identical in law, the mean duration of the observed idle period is

$$
\mathrm{E}\left[d(0)-g(0) \mid Q_{0}=0\right]=-2 \frac{\Phi_{Y}(0) \psi_{Y}(1)}{1-\Phi_{Y}(0)} F^{\prime}(0) .
$$


Note that, using the function $F$, we may write, from Proposition 7 ,

$$
\mathrm{E}_{g}\left[\mathrm{e}^{-\alpha d(0)}\right]=1-\alpha F(\alpha)
$$

and

$$
\mathrm{E}_{g}[d(0)]=F(0)
$$

Now, by the 'inspection paradox',

$$
\mathrm{E}_{g}[d(0)] \leq \mathrm{E}\left[d(0)-g(0) \mid Q_{0}=0\right],
$$

which, after some manipulations, is equivalent to

$$
\left(1-\Phi_{Y}(0)\right)^{2} \leq 2\left(\Phi_{Y}(0)^{2}-\Phi_{Y}(0)+\Phi_{Y}^{\prime}(0) \psi_{Y}(1)\right) .
$$

Example 5. (Continuation of Example 1.) Consider $Y_{t}=\sigma B_{t}-\mu t$, and assume that $0<\mu<1$. Here the rate of beginnings of idle (or busy) periods is

$$
\lambda=\psi_{Y}^{\prime}(0) \theta^{*}=\frac{2 \mu(1-\mu)}{\sigma^{2}} .
$$

The mean duration of a typical idle period of $Q$ is $\sigma^{2} / 2 \mu$, while the mean duration of a typical busy period of $Q$ is $\sigma^{2} / 2(1-\mu)$. To find, for example, the distribution of a typical busy period, we use Proposition 8. We have, see (21),

$$
\psi_{\Lambda}(q)=q-\Phi_{Y}(q)
$$

where $\Phi_{Y}$ is the inverse function of $\psi_{Y}$, i.e.

$$
\Phi_{Y}(q)=\frac{\sqrt{\mu^{2}+2 \sigma^{2} q^{2}}-\mu}{\sigma^{2}},
$$

and $\Phi_{\Lambda}$ is the inverse function of $\psi_{\Lambda}$, i.e.

$$
\Phi_{\Lambda}(\alpha)=\frac{(1-\mu)+2 \sigma^{2} \alpha+\sqrt{(1-\mu)^{2}+4 \sigma^{2} \alpha}}{2 \sigma^{2}},
$$

and so the Laplace transform of the typical busy period is

$$
\mathrm{E}_{d}\left[\mathrm{e}^{-\alpha g(1)}\right]=\frac{(1-\mu)+\sqrt{(1-\mu)^{2}+4 \sigma^{2} \alpha}}{(1-\mu)+2 \sigma^{2} \alpha+\sqrt{(1-\mu)^{2}+4 \sigma^{2} \alpha}} .
$$

\section{Appendix A. On Skorokhod reflection, fluid queues, and stationarity}

In this appendix we review some facts about the Skorokhod reflection of a process with stationary ergodic increments. We carefully define the system, give conditions for its stability, and recall some distributional relations based on Palm calculus; see [1, Lemma 3.1.1] and [15]. Although the setup is much more general than the one used in this paper for concrete calculations, it is nevertheless interesting to isolate those properties that are not based on specific distributional assumptions (such as the Markovian property or independent increments), but are consequences of the more general stationary framework. 
Let $(\Omega, \mathcal{F}, \mathrm{P})$ be a probability space together with a P-preserving flow $\left(\theta_{t}, t \in \mathbb{R}\right)$. That is, for each $t \in \mathbb{R}, \theta_{t}: \Omega \rightarrow \Omega$ is measurable with measurable inverse, $\theta_{0}$ is the identity function, $\theta_{t} \circ \theta_{s}=\theta_{s+t}$ for all $s, t \in \mathbb{R}$, and $\mathrm{P}\left(\theta_{t} A\right)=\mathrm{P}(A)$ for all $t \in \mathbb{R}, A \in \mathcal{F}$. Consider a process $\mathrm{W}=\left(\mathrm{W}_{t}, t \in \mathbb{R}\right)$ satisfying $\left(\mathrm{W}_{t}-\mathrm{W}_{s}\right) \circ \theta_{u}=\mathrm{W}_{t+u}-\mathrm{W}_{s+u}$ for all $s, t, u \in \mathbb{R}$ and all $\omega \in \Omega$. Then $\mathrm{W}$ has stationary increments. We let $\mathrm{E}$ denote the expectation with respect to $\mathrm{P}$.

Following [15], we define the SDS driven by $\mathrm{W}$ as a two-parameter stochastic flow:

$$
\begin{aligned}
\mathcal{R}_{s, t} \mathrm{~W}(x): & =\left[x+\mathrm{W}_{t}-\mathrm{W}_{s}\right]-\inf _{s \leq u \leq t}\left\{\left(x+W_{s}-W_{u}\right) \wedge 0\right\} \\
& =\sup _{s \leq u \leq t}\left(\mathrm{~W}_{t}-\mathrm{W}_{u}\right) \vee\left(x+\mathrm{W}_{t}-\mathrm{W}_{s}\right), \quad x \geq 0, s \leq t .
\end{aligned}
$$

Thus, for each $s<t$, we have a random element $\mathcal{R}_{s, t} \mathrm{~W}$ taking values in a space of continuous functions. The family $\left(\mathcal{R}_{s, t} \mathrm{~W},-\infty<s<t<\infty\right)$ is a stochastic flow because the following composition rule (semigroup property) holds for each $\omega \in \Omega$ :

$$
\begin{gathered}
\mathcal{R}_{s, t} \mathrm{~W}=\mathcal{R}_{u, t} \mathrm{~W} \circ \mathcal{R}_{s, u} \mathrm{~W}, \quad s \leq u \leq t, \\
\mathcal{R}_{t, t} \mathrm{~W}(x)=x, \quad t \in \mathbb{R}, \quad x \geq 0 .
\end{gathered}
$$

It is a stationary stochastic flow because, for each $x \in \mathbb{R}_{+}$, we have

$$
\mathcal{R}_{s, t} \mathrm{~W}(x) \circ \theta_{u}=\mathcal{R}_{s+u, t+u} \mathrm{~W}(x), \quad-\infty<s \leq t<\infty, u \in \mathbb{R} .
$$

We say that the process $Z=\left(Z_{t}, t \in \mathbb{R}\right)$ constitutes a stationary solution of the SDS driven by $W$ if $Z$ is $W$-measurable and if

$$
\mathrm{Z}_{t}=\mathcal{R}_{s, t} \mathbf{W}\left(\mathbf{Z}_{s}\right), \quad s \leq t, \quad \mathrm{Z}_{t} \circ \theta_{u}=\mathbf{Z}_{t+u}, \quad t, u \in \mathbb{R} .
$$

Existence and uniqueness are guaranteed under some assumptions. Lemma 8 and its corollary, below (formulated as Lemma 1 in [15]), are proved in [12] (see also [14]).

Lemma 8. Assume that $\sup _{-\infty<s \leq 0} \mathrm{~W}_{s}<\infty$ and that $\underline{\lim }_{t \rightarrow \infty} \mathrm{W}_{t}<\infty$, P-a.s. Then there is a unique stationary solution to the SDS driven by $\mathrm{W}$. This is given by

$$
\mathrm{Z}_{t}=\sup _{-\infty<u \leq t}\left(\mathrm{~W}_{t}-\mathrm{W}_{u}\right)=: \widetilde{\mathcal{R}}_{t} \mathbf{W} .
$$

Quite often, in addition to stationarity of the flow, we also assume ergodicity, namely, that each $A \in \mathcal{F}$ that is invariant under $\theta_{t}$ for all $t$ has $\mathrm{P}(A)$ equal to 0 or 1 . Owing to Birkhhoff's individual ergodic theorem, Lemma 8 immediately yields the following corollary.

Corollary 2. Under the ergodicity assumption, and if $\mathrm{E}\left[\mathrm{W}_{1}\right]<0$, there is a unique stationary solution $\mathrm{Z}$ to the SDS driven by $\mathrm{W}$. The process $\mathrm{Z}$ is given by (45) and $\mathrm{Z}$ is an ergodic process.

For the purposes of this paper, assume that $\mathrm{W}$ is of the form

$$
\mathrm{W}_{t}-\mathrm{W}_{s}=M(s, t]-\beta(t-s), \quad s \leq t,
$$

where $M$ is a locally finite stationary random measure and

$$
0<\alpha:=\mathrm{E}[M(0,1)]<\beta .
$$

Let $\mathrm{P}_{M}$ be the Palm probability (see [9] and [10, Chapter 11]) with respect to $M$ :

$$
\mathrm{P}_{M}(C)=\frac{1}{\alpha} \mathrm{E}\left[\int_{(0,1]} \mathbf{1}_{C} \circ \theta_{t} M(\mathrm{~d} t)\right] .
$$

The following lemma is a consequence of Theorem 3 of [15]. 
Lemma 9. (Distributional Little's law [15, Theorem 3].) Let Z be the unique stationary solution to the SDS driven by $\mathrm{W}$ of the form (46). Assume that (47) holds. Then, for any measurable function $\psi:[0, \infty) \rightarrow[0, \infty)$, we have

$$
\mathrm{E}\left[\psi\left(Z_{0}\right)\right]=\left(1-\frac{\alpha}{\beta}\right) \psi(0)+\frac{\alpha}{\beta} \mathrm{E}_{M}\left[\frac{\int_{\mathrm{Z}_{0-}}^{\mathrm{Z}_{0}} \psi(x) \mathrm{d} x}{\mathrm{Z}_{0}-\mathrm{Z}_{0-}}\right],
$$

with the understanding that the last fraction on the right-hand side equals $\psi\left(Z_{0}\right)$ on the event $\left\{Z_{0}=Z_{0-}\right\}$.

It should be noted that the decomposition of W, (46), is not unique; nevertheless, (48) holds, regardless of which decomposition of $\mathrm{W}$ we choose.

\section{Appendix B. Exit times for spectrally negative Lévy processes}

In this section we consider a spectrally negative Lévy process and some facts regarding the first time the process exits an unbounded interval. Let $Y=\left(Y_{t}, t \in \mathbb{R}\right)$ be a spectrally negative Lévy process with Lévy measure $\Pi$. In other words, let $B$ be a standard Brownian motion, let $\eta$ be an independent Poisson random measure on $\mathbb{R} \times \mathbb{R}_{-}$such that

$$
\mathrm{E}[\eta(\mathrm{d} t, \mathrm{~d} y)]=\mathrm{d} t \Pi(\mathrm{d} y), \quad \Pi\{0\}=0, \quad \int_{\mathbb{R}_{-}}\left(y^{2} \wedge 1\right) \Pi(\mathrm{d} y)<\infty,
$$

let $a \in \mathbb{R}, \sigma \geq 0$, and define, for $-\infty<s \leq t<\infty$,

$$
\begin{aligned}
Y(s, t]= & a(t-s)+\sigma\left(B_{t}-B_{S}\right) \\
& +\int_{(s, t]} \int_{(-\infty,-1]} y \eta(\mathrm{d} u, \mathrm{~d} y)+\int_{(s, t]} \int_{(-1,0)} y(\eta(\mathrm{d} u, \mathrm{~d} y)-\mathrm{d} u \Pi(\mathrm{d} y)) .
\end{aligned}
$$

Note that we have thus defined only the increments of $Y$. (The reason that the increments are more fundamental than the process itself is amply explained in [24].) If we set

$$
Y_{t}:=\left\{\begin{array}{ll}
Y(0, t], & t \geq 0, \\
-Y(t, 0], & t<0,
\end{array} \quad t \in \mathbb{R}\right.
$$

we have

$$
Y(s, t]=Y_{t}-Y_{s}, \quad-\infty<s \leq t<\infty .
$$

The choice $Y_{0}=0$ is a convenient assumption (note that the stationary process $\widetilde{\mathcal{R}}_{t} Y$ of interest in this paper depends only the increments of $Y$ ). (In the case that $\int_{-1}^{0}|y| \Pi(\mathrm{d} y)<\infty$ and $\sigma=0$, the process $Y$ has bounded variation paths and can also be represented as

$$
Y(s, t]=d_{Y}(t-s)+\int_{(s, t]} \int_{(-\infty, 0]} y \eta(\mathrm{d} u, \mathrm{~d} y)
$$

for some constant $d_{Y}$ known as the drift of $Y$.)

To be more precise, especially for the construction of the stationary versions of processes in this paper, we introduce shifts. Assume that $(B, \eta)$ is defined on a probability space $(\Omega, \mathcal{F}, \mathrm{P})$ taken, without loss of generality, to be the canonical space $\Omega=C(\mathbb{R}) \times \mathcal{N}\left(\mathbb{R}^{2}\right)$, where $C(\mathbb{R})$ is the set of the continuous functions on $\mathbb{R}$ and $\mathcal{N}\left(\mathbb{R}^{2}\right)$ is the set of integer-valued measures 
on $\mathbb{R}^{2}$. (The space $\mathcal{N}\left(\mathbb{R}^{2}\right)$ is endowed with the topology of weak convergence; see, e.g. [10, Chapter 15].) Let $\mathrm{P}$ be the product measure on the Borel sets of $C(\mathbb{R}) \times \mathcal{N}\left(\mathbb{R}^{2}\right)$ that makes $B$ a standard Brownian motion and $\eta$ a Poisson random measure with mean measure as in (49), and to each $\omega=(\varphi, \mu) \in C(\mathbb{R}) \times \mathcal{N}\left(\mathbb{R}^{2}\right)$, let $B(\varphi, \mu)=\varphi$ and $\eta(\varphi, \mu)=\mu$. Consider also the natural shift $\left(\theta_{t}, t \in \mathbb{R}\right)$ on $\Omega$ defined by

$$
\theta_{t}(\varphi, \mu)(s, A)=(\varphi(t+s), \mu(A+s)), \quad s \in \mathbb{R}, A \in \mathcal{B}\left(\mathbb{R}^{2}\right),
$$

where $A+s:=\left\{(t+s, y) \in \mathbb{R}^{2}:(t, y) \in A\right\}$. By construction, $Y$ has càdlàg paths (i.e. paths that are continuous from the right with left limits), and, under $\mathrm{P}$, it has stationary (and independent) increments. Henceforth, we shall denote by $\mathrm{P}_{x}$ the conditional probability of $\mathrm{P}$ given $Y_{0}=x$ and by $\mathrm{E}_{x}$ the expectation with respect to it.

All of the following facts are standard results which can be found, for example, in [2, Chapter VI] and [18, Chapter 8]. See also [19] for a review which is more convenient for the setting at hand. Let $\Psi_{Y}: \mathbb{R} \rightarrow \mathbb{C}$ denote the characteristic exponent of $Y$, i.e.

$$
\mathrm{E}\left[\exp \left\{\mathrm{i} \theta Y_{1}\right\}\right]=\exp \left\{-\Psi_{Y}(\theta)\right\}, \quad \theta \in \mathbb{R},
$$

and let $\psi_{Y}:[0, \infty) \mapsto \mathbb{R}$ denote the Laplace exponent of $Y$, i.e.

$$
\psi_{Y}(\beta)=\log \mathrm{E}\left[\exp \left\{\beta Y_{1}\right\}\right], \quad \beta \geq 0 .
$$

It is well known that $\psi_{Y}$ is infinitely differentiable, strictly convex, $\psi(0)=0, \lim _{\beta \rightarrow \infty} \psi_{Y}(\beta)=$ $\infty$, and that

$$
\psi_{Y}^{\prime}(0+)=\mathrm{E}\left[Y_{1}\right]=\mathrm{E}\left[Y_{t+1}-Y_{t}\right] \in \mathbb{R} \cup\{-\infty\} .
$$

For each $q \geq 0$, let

$$
\Phi_{Y}(q)=\sup \left\{\beta \geq 0: \psi_{Y}(\beta)=q\right\} .
$$

Since $Y$ drifts to $\infty$, oscillates, and drifts to $-\infty$ according to whether $\psi_{Y}^{\prime}(0+)>0, \psi_{Y}^{\prime}(0+)=$ 0 , and $\psi_{Y}^{\prime}(0+)<0$, respectively, it follows that $\Phi_{Y}(0)>0$ if and only if $\psi_{Y}^{\prime}(0+)<0$ and otherwise $\Phi_{Y}(0)=0$. It is also easy to see that $\Phi_{Y}(q)>0$ for all $q>0$.

Define also the scale functions $W^{(q)}(x)$ and $Z^{(q)}(x)$ via their Laplace transforms

$$
\begin{gathered}
\int_{0}^{\infty} \mathrm{e}^{-\beta x} W^{(q)}(x) \mathrm{d} x=\frac{1}{\psi_{Y}(\beta)-q}, \\
\int_{0}^{\infty} \mathrm{e}^{-\beta x} Z^{(q)}(x) \mathrm{d} x=\frac{\psi_{Y}(\beta)}{\beta\left(\psi_{Y}(\beta)-q\right)},
\end{gathered}
$$

defined for all $\beta>\Phi_{Y}(q)$. The functions $\Phi_{Y}, W^{(q)}$, and $Z^{(q)}$ appear in the expressions for the Laplace transform of the first passage times,

$$
\begin{aligned}
\tau_{x}^{+} & :=\inf \left\{t>0: Y_{t}>x\right\}, \\
\tau_{-x}^{-} & :=\inf \left\{t>0: Y_{t}<-x\right\},
\end{aligned}
$$

as follows (cf. [18, Theorem 8.1, p. 214]).

Lemma 10. For all $q \geq 0$ and $x \geq 0$,

$$
\begin{gathered}
\mathrm{E}\left[\exp \left\{-q \tau_{x}^{+}\right\}\right]=\exp \left\{-\Phi_{Y}(q) x\right\}, \\
\mathrm{E}\left[\exp \left\{-q \tau_{-x}^{-}\right\}\right]=Z^{(q)}(x)-\frac{q}{\Phi_{Y}(q)} W^{(q)}(x) .
\end{gathered}
$$




\section{References}

[1] Baccelli, F. And Brémaud, P. (2003). Elements of Queueing Theory. Palm Martingale Calculus and Stochastic Recurrences (Appl. Math. 26), 2nd edn. Springer, Berlin.

[2] Bertoin, J. (1996). Lévy Processes. Cambridge University Press.

[3] Bingham, N. H. (1975). Fluctuation theory in continuous time. Adv. Appl. Prob. 7, 705-766.

[4] Blumenthal, R. M. and Getoor, R. (1968). Markov Processes and Potential Theory. Academic Press, New York.

[5] Borodin, A. N. and SAlminen, P. (2002). Handbook of Brownian Motion-Facts and Formulae, 2nd edn. Birkhäuser, Basel.

[6] Distributed Computing Industry Weekly Newsletter, Vol. XXII, Issue 8, June 30, 2008. Available at http://www. dcia.info/news/newsletter_2008-06-30.htm.

[7] FristedT, B. E. (1974). Sample functions of stochastic processes with stationary independent increments. In Adv. Prob. 3, eds P. Ney and S. Port, Dekker, New York, pp. 241-396.

[8] Harrison, J. M. (2003). A broader view of Brownian networks. Ann. Appl. Prob. 13, 1119-1150.

[9] Kallenberg, O. (1983). Random Measures, 3rd edn. Academic Press, London.

[10] Kallenberg, O. (2002). Foundations of Modern Probability, 2nd edn. Springer, New York.

[11] Kella, O. (1993). Parallel and tandem fluid networks with dependent Lévy inputs. Ann. Appl. Prob. 3, 682-695.

[12] Kella, O. (1997). Stochastic storage networks: stationarity and the feedforward case. J. Appl. Prob. 34, 498-507.

[13] Kella, O. ANd Whitt, W. (1992). A tandem fluid network with Lévy input. In Queueing and Related Models, eds I. Basawa and U. N. Bhat, Oxford University Press, pp. 112-128.

[14] Kella, O. ANd Whitt, W. (1996). Stability and structural properties of stochastic storage networks. J. Appl. Prob. 33, 1169-1180.

[15] Konstantopoulos, T. and Last, G. (2000). On the dynamics and performance of stochastic fluid systems. $J$. Appl. Prob. 37, 652-667.

[16] Konstantopoulos, T., Zazanis, M. and de Veciana, G. (1997). Conservation laws and reflection mappings with an application to multiclass mean value analysis for stochastic fluid queues. Stoch. Process Appl. 65, 139-146.

[17] Kozlova, M. and Salminen, P. (2004). Diffusion local time storage. Stoch. Process Appl. 114, 211-229.

[18] Kyprianou, A. (2006). Introductory Lectures on Fluctuations of Lévy Processes with Applications. Springer, Berlin.

[19] Kyprianou, A. ANd Palmowski, Z. (2004). A martingale review of some fluctuation theory for spectrally negative Lévy processes. In Séminaire de Probabilités XXXVIII (Lecture Notes Math. 28). Springer, Berlin, pp. 16-29.

[20] Mannersalo, P., Norros, I. and Salminen, P. (2004). A storage process with local time input. Queueing Systems 46, 557-577.

[21] Pitman, J. (1986). Stationary excursions. In Séminaire de Probabilités XXI(Lecture Notes Math. 1247). Springer, Berlin, pp. 289-302.

[22] Salminen, P. (1993). On the distribution of diffusion local time. Statist. Prob. Lett. 18, 219-225.

[23] SiRviö, M. (2006). On an inverse subordinator storage. Helsinki University of Technology, Inst. Math. Rep. Series A501.

[24] Tsirelson, B. (2004). Nonclassical stochastic flows and continuous products. Prob. Surveys 1, 173-298.

[25] Zolotarev, V. M. (1964). The first-passage time of a level and the behaviour at infinity for a class of processes with independent increments. Theory Prob. Appl. 9, 653-664. 